\title{
Stochastic Resonance with a Joint Woods-Saxon and Gaussian Potential for Bearing Fault Diagnosis
}

\author{
Haibin Zhang, Qingbo He, Siliang Lu, and Fanrang Kong \\ Department of Precision Machinery and Precision Instrumentation, University of Science and Technology of China, Hefei, \\ Anhui 230026, China \\ Correspondence should be addressed to Qingbo He; qbhe@ustc.edu.cn
}

Received 17 April 2014; Revised 11 May 2014; Accepted 16 May 2014; Published 9 June 2014

Academic Editor: Xuefeng Chen

Copyright (c) 2014 Haibin Zhang et al. This is an open access article distributed under the Creative Commons Attribution License, which permits unrestricted use, distribution, and reproduction in any medium, provided the original work is properly cited.

\begin{abstract}
This work aims for a new stochastic resonance (SR) model which performs well in bearing fault diagnosis. Different from the traditional bistable SR system, we realize the SR based on the joint of Woods-Saxon potential (WSP) and Gaussian potential (GP) instead of a reflection-symmetric quartic potential. With this potential model, all the parameters in the Woods-Saxon and Gaussian SR (WSGSR) system are not coupled when compared to the traditional one, so the output signal-to-noise ratio (SNR) can be optimized much more easily by tuning the system parameters. Besides, a smoother potential bottom and steeper potential wall lead to a stable particle motion within each potential well and avoid the unexpected noise. Different from the SR with only WSP which is a monostable system, we improve it into a bistable one as a general form offering a higher SNR and a wider bandwidth. Finally, the proposed model is verified to be outstanding in weak signal detection for bearing fault diagnosis and the strategy offers us a more effective and feasible diagnosis conclusion.
\end{abstract}

\section{Introduction}

Stochastic resonance (SR) has been firstly introduced by Benzi and coworkers [1] to explain the more or less periodic occurrence of earth's ice ages. We know the main effect of SR is to enhance the response of a bistable system to weak periodic driving by the injection of random noise [2]. As researchers found that the phenomenon of SR benefits weak signal detection under the background noise, during the past three decades, SR being one of the most exciting nonlinear phenomena has attracted considerable attentions in a wide range of research [3-9]. Via the SR, the output signal of a nonlinear dynamic system can be enhanced with optimized signal-tonoise ratio (SNR) by means of noise addition to the system.

On the other hand, rotating machinery plays a significant role in a wide range of industrial applications, such as transportation vehicles, aeroengine, and power generators [7], while rolling bearing works as a necessary part for rotating machine. Therefore safe and reliable operation of a rolling bearing is an important guarantee to reduce economic losses and avoid personal injury. As a result, accurate health monitoring and diagnosis system is of great significance to indicate the incipient fault that may occur in a rotating machine [10].
In the past decades, many algorithms for this particular application have been proposed [11]. The most common methods are based on signal analysis technique to extract the useful features from the original signals acquired by kinds of sensors. However, the fault signal of the rolling bearing is often overwhelmed with heavy background noise coming from other coupled machine components and working environment, which makes some incipient faults not easy to be recognized. The challenge of fault recognition requires enhancing the weak fault information from heavy background noise.

Noise filtering seems a common and effective method which can suppress the noise and improve the output signalto-noise ratio (SNR), while the SR offers us a new approach and idea. As compared to traditional techniques that mainly focus on how to suppress the noise, the SR has the merit of signal enhancement by the aid of the noise which means the noise plays an active role in our work [2]. During the development of SR in signal enhancement, many researches focus on some issues referred to next. For the restriction of small parameter, several system parameter tuning or noise intensity tuning methods have been proposed to make them more adaptive, such as frequency shifted and rescaling $[3,4]$, modulation and demodulation [5], multiscale noise tuning 
$[7,8,12]$, normalized scale transform [13], and adaptive stepchanged [14]. Besides, SR-based signal processing could be improved for a better performance with target of higher SNR or SNRI. Cascaded bistable system was proposed by He et al. [15], which connects two or more bistable systems in series. $\mathrm{Li}$ et al. studied the SR effects with multistable potential model [6]. Zhang et al. referred to a multiscale bistable stochastic resonance array [9], while most of current SR researches focus on the traditional bistable potential model with a reflectionsymmetric quartic potential. Lu et al. firstly extended the application of Woods-Saxon stochastic resonance (WSSR) which is a monostable SR system to the weak signal detection area [16]. Motivated by this work, we propose a new potential model that maintains the advantages of the WSSR to improve the output SNR and broaden the bandwidth of characteristic frequency and noise intensity.

In this paper, we change the WSSR from monostable into bistable by the cooperation of a Gaussian potential (GP). The WSP was firstly put into use by Deza et al. for wide spectrum energy harvesting based on the SR principle [17]. It is a heuristic potential with three parameters which reproduces the qualitative features of the mean force exerted on each nucleon inside the atomic nucleus. The WSP is monostable, and it will show different shapes with different potential height, width, and wall steepness by tuning its system parameters. Different shapes of WSP in SR system can yield different outputs for the same input signal $[16,17]$. Gaussian potentials have been used extensively in nuclear physics as a basis for twobody interactions firstly [18-20]. Later, researchers found its favorable application in solution of differential equation especially the solution of Schrodinger equation. Stephenson applied it to the calculation of the eigenvalues of the threedimensional Schrodinger equation with an attractive radial GP [21]. Eigenvalues and approximate eigenfunctions of the Schrodinger equation with an attractive radial GP are obtained from a first-order perturbation treatment based on a scaled harmonic oscillator model by Cohen [22]. A GP clearly satisfies the conditions of symmetry, continuity, and confining. This model potential is smooth and possesses a finite depth as well as a characteristic finite radius. As a result of these characteristics, we set a GP working together with WSP where the GP acts as a potential barrier that will change the monostable WSSR into a bistable Woods-Saxon and Gaussian SR (WSGSR) system. The SR effect comes up much more easily with the help of noise and a higher output SNR than we can get.

The rest of the paper is arranged as follows. In Section 2, we introduce the framework of the model of WSP and GP as well as the way they unite together as a bistable model. Section 3 shows us the WSGSR system driven by the joint potential. It gives the criterion for evaluation and the steps to realize weak signal detection based on WSGSR. In Section 4, we utilize the simulated bearing fault signals to evaluate the proposed WSGSR system in comparison with traditional bistable SR, also the WSSR. In Section 5, experimental studies by some practical defective bearing signals are conducted to confirm the effectiveness of the proposed system and method and this reveals the engineering application of the proposed model. Finally, conclusions are drawn in Section 6.

\section{Potential Model}

2.1. Woods-Saxon Potential. In WSP model, the potential function $U_{\mathrm{WS}}(x)$ is a symmetric nonlinear potential. Its form was proposed in the midfifties by Woods and Saxon [23] and firstly used in the harvest of energy by Deza et al. [17] as a mean-field potential for single-nucleon states within the shell model of nuclear structure which can be expressed as follows:

$$
U_{\mathrm{WS}}(x)=-\frac{V_{1}}{1+\exp \left(\left(|x|-R_{1}\right) / a\right)} .
$$

Here parameter $V_{1}$ affects the depth of the potential and parameter $R_{1}$ works on the width of the potential while parameter $a$ determines the wall steepness of the potential. This potential with three parameters reproduces the qualitative features of the mean force exerted on each nucleon inside the atomic nucleus. Figure 1 shows us the effects that the three parameters have on the potential distribution with the changing of $x$. The black curves show the potential $U_{\mathrm{WS}}(x)$ at fixed $V_{1}=2, R_{1}=1$, and varying $a$ from 0.02 to 0.2 . The result tells us that the value $a$ will affect the plainness of the potential well. It will become flatter with a smaller $a$ and even a square well with width of $2 R_{1}$ for $a=0$. As $a$ grows the potential walls may become smoother, maintaining the value $-V_{1} / 2$ at $|x|=R_{1}$. The blue curves indicate the influence of $V_{1}$ while the red ones reveal that of $R_{1} . V_{1}$ plays an effect on the depth and $R_{1}$ works on the width of the well. Hence the potential shape can be adjusted by parameters $V_{1}, R_{1}$, and $a$ separately or jointly.

2.2. Gaussian Potential. The attractive radial Gaussian potential of the form

$$
U_{G}(x)=-V_{2} \exp \left(-\frac{x^{2}}{R_{2}^{2}}\right)
$$

is of importance and has been used extensively in nuclear physics during the past decades. As a confining potential, GP has a behavior for large $x$ laid down rapidly [18]. Here $V_{2}$ works on the depth of the potential well and $R_{2}$ is the range of the confinement potential, which corresponds to the radius. Figure 2 shows us the potential distribution as well as the effects of the parameters. We can find that the potential well with depth of $V_{2}$ converges to 0 rapidly on both the side and the smaller $R_{2}$, the steeper potential walls.

2.3. Joint Woods-Saxon and Gaussian Potential. With the two introduced models, we proposed a new bistable potential model WSG by the combination of WSP and GP which can be expressed as follows:

$$
\begin{aligned}
U(x)=U_{\mathrm{WS}}(x)-U_{G}(x)= & -\frac{V_{1}}{1+\exp \left(\left(|x|-R_{1}\right) / a\right)} \\
& +V_{2} \exp \left(-\frac{x^{2}}{R_{2}^{2}}\right) .
\end{aligned}
$$

Its potential distribution is shown in Figure 3 with parameters: $V_{1}=2, R_{1}=1, a=0.02, V_{2}=1$, and $R_{2}=0.05$. 


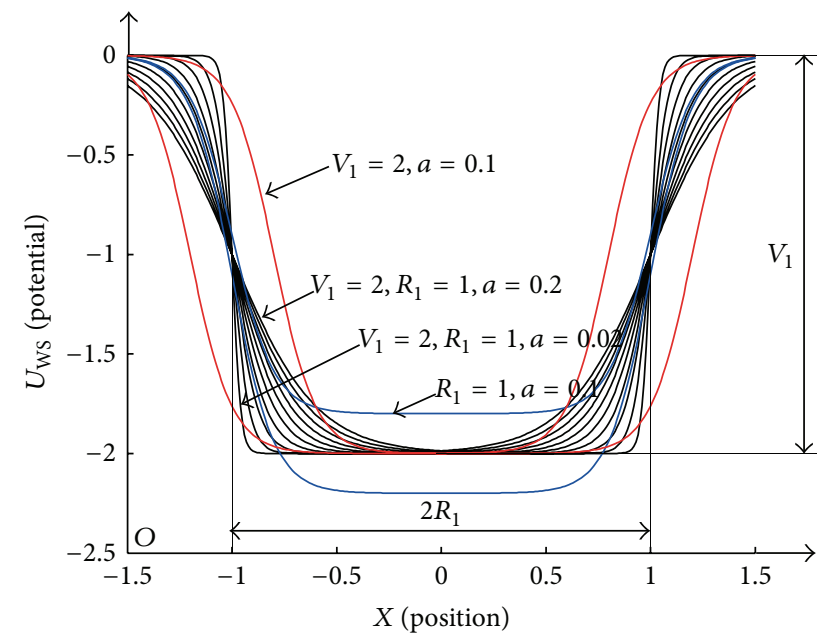

FIGURE 1: Shape of WSP $U_{\text {WS }}(x)$ with different parameters. Black curves for different $a$, blue curves for different $V_{1}$, and red curves for different $R_{1}$.

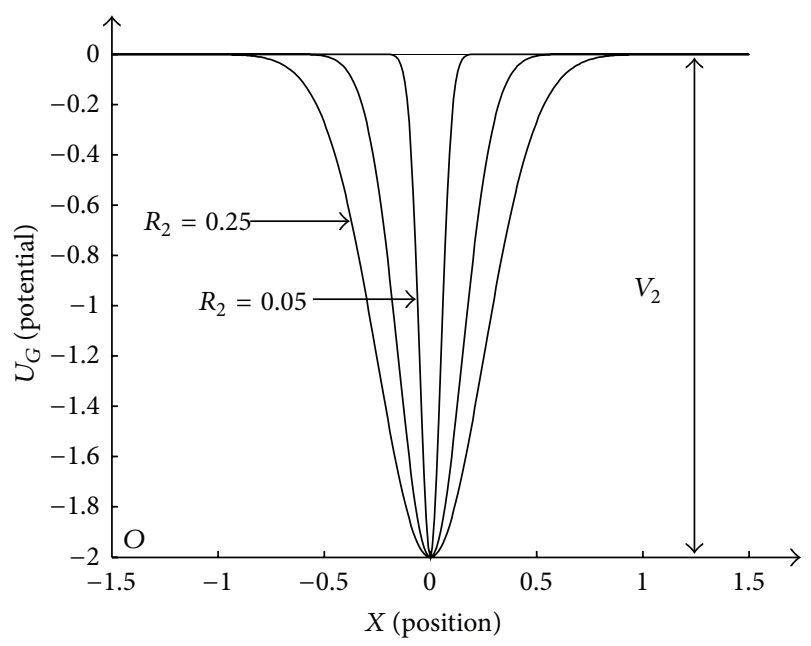

FIgURE 2: Shape of GP $U_{G}(x)$ with different $R_{2}$, where $V_{2}=2$.

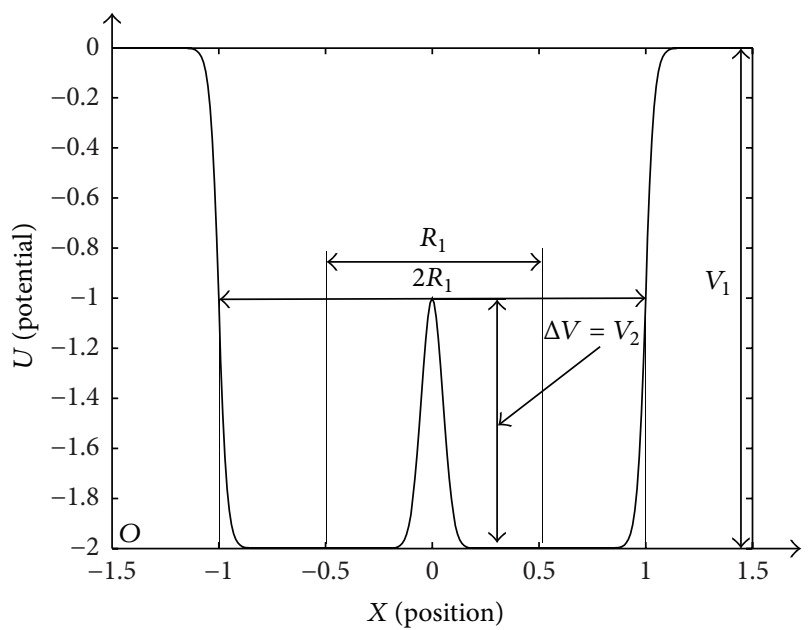

FIGURE 3: Shape of WSGP $U(x)$ with $V_{1}=2, R_{1}=1, a=0.02$, $V_{2}=1$, and $R_{2}=0.05$.
We can find a novel bistable potential model where $U(x)$ has two symmetric potential wells with depth of $V_{2}$ indicating the barrier height. The width of each well and the distance between the two wells can be adjusted by $R_{1}$ and $R_{2}$. So the shape of the bistable potential model can be tuned by each parameter independently. The combination of two models works as a mathematical model and the adding of GP makes the monostable WSP turn to a bistable WSGP. The result aids in gaining a new model that contains the superiority of both traditional bistable SR and WSSR. It is still obvious that the potential of the WSG possesses flatter well and steeper barrier whose characteristics will contribute to better performance of SR. Maybe there are some other combinations while they might not offer a bistable model or have the both advantages. Besides, the new model can change easily between the monostable and bistable states, which indicates that (3) works as a more general form. And with this mathematical form, we can process the signal with a novel SR model to extract the weak periodic component that will be introduced later.

\section{Stochastic Resonance System}

3.1. WSGSR Model. The three basic ingredients of producing SR phenomenon are (1) a bistable or multistable system, (2) a weak coherent input (such as a periodic signal), and (3) a source of noise that is inherent in the system or that adds to the coherent input. Considering the overdamped motion of a Brownian particle in a bistable potential in the presence of noise and periodic forcing, a SR system can be expressed as follows:

$$
\frac{d x}{d t}=-V^{\prime}(x)+A_{0} \sin \left(2 \pi f_{0} t+\varphi\right)+n(t)
$$

where $V(x)$ denotes the reflection-symmetric quartic potential

$$
V(x)=-\frac{a}{2} x^{2}+\frac{b}{4} x^{4}
$$

In (4), we set $n(t)=\sqrt{2 D} \xi(t)$ with $\langle n(t) n(t+\tau)\rangle=$ $2 D \delta(t)$, where $D$ is the noise intensity and $\xi(t)$ presents a zero mean unit variance Gaussian white noise. $a$ and $b$ are real parameters. $A_{0}$ is the periodic signal amplitude and $f_{0}$ is the modulation frequency. In this case, (4) can be written as follows:

$$
\frac{d x}{d t}=a x-b x^{3}+A_{0} \sin \left(2 \pi f_{0} t+\varphi\right)+\sqrt{2 D} \xi(t) .
$$

Equation (6) indicates the nonlinear Langevin equation for one variable $x$ with a traditional reflection-symmetric quartic potential and forms the common bistable SR model. If we change the quartic potential model to the WSGP one, it will be the WSGSR system. Substituting (3) to (4), the new function of SR with WSGP will be in form

$$
\begin{aligned}
\frac{d x}{d t}= & -\frac{V_{1}}{a} \operatorname{sgn}(x) \exp \left(\frac{|x|-R_{1}}{a}\right)\left(1+\exp \left(\frac{|x|-R_{1}}{a}\right)\right)^{-2} \\
& +\frac{2 V_{2} x}{R_{2}^{2}} \exp \left(-\frac{x^{2}}{R_{2}^{2}}\right)+A_{0} \sin \left(2 \pi f_{0} t+\varphi\right)+n(t),
\end{aligned}
$$


where $\operatorname{sgn}(x)$ denotes the sign function. As the WSGP showed in Figure 3, then what is the difference between the systems indicated by (6) and (7), respectively? It can be seen from (7) that the system output $x$ (left hand side of (7)) is determined by the potential (first two items of right hand side of (7)) and the input signal (third and last items of the right hand side of (7)). The only difference between the two systems is the potential model.

In fact, the effect of SR is the result of the joint action of the system, input signal, and random noise. We build a specific scene that the particle oscillates within the potential under the collective excitation from the potential force, the periodic force, and the noise force to describe SR phenomenon concretely. Among all the kinds of force, the potential force is generated by the gradient of the potential curve which can be described as the first-order derivative of $U(x)$. In practical situation, periodic force and noise force may be constant, so the SR system efficiency will be greatly affected by the potential force to a large extent.

Generally speaking, for the shape of the driving potential such as (3) or (5), if the potential wells are too wide, the particle could not reach the potential wall or the barrier that it always needs to provide the maximal restoring force in one periodic excitation cycle, and hence the restoring force will be helpless in enhancing the particle periodic motion. On the other hand, with too narrow wells, the particle may not have the opportunity to reach the expected destination in the right direction within one excitation cycle because the potential wall may have already forced the particle to move backward prematurely. Similarly, a too high potential barrier will make it difficult for the particle to transmit through the two wells with a periodic input signal of small energy and it may just move among one of the wells. So it cannot work to amplify the small input signal from heavy background noise. But if the barrier height is too low, just the noise force can make the particle traverse without the periodicity. In this case, the particle which means the output oscillates disorderedly and it does not coincide to what we expect. Besides, a steep potential wall will lead to an intense restoring force, which may further cause the particle to be rebounded rapidly. However, when the restoring speed is too high, it may lead to a counteractive effect as the periodic oscillation could not keep pace with the restoring speed. On the contrary, if the potential wall is too gradual, it may not provide enough acceleration to enhance the periodic oscillation. Thus, these indicate that only when the potential is in an optimal condition that can match the periodic force, the periodic and the noise forces could have the effect to amplify the particle oscillation and the periodic signal can then be enhanced.

With these features, we can find the advantages of the WSGSR over the traditional one qualitatively. In order to make the potential model in an optimal status, we need to adjust the parameters. For the traditional potential model as in (5), the parameters $a$ and $b$ work interactively. That is to say, it is not convenient to adjust one isolate potential feature (e.g., barrier height $a^{2} / 4 b$ ) while keeping the other features (e.g., equilibrium positions $\pm \sqrt{a / b}$ ) invariable by adjusting the parameters. Assuming that the barrier height has been adjusted to the optimal condition, the equilibrium positions or the potential wall steepness may not be in the optimal conditions and still the system is difficult to be tuned to an optimal status. While, for the WSGP as (3) showed in Figure 3, we find the parameters work on the potential features independently, that means we can adjust one of the features to an optimal condition by tuning the parameters without disturbing the others. For example, the barrier height is affected by $V_{2}$ only. Note that when $V_{2}=0$, it performs as a WSSR system [16]. So the proposed SR model can be regarded as a common form with WSSR as its specific case. We can also tune $a$ and $R_{2}$ to adjust the steepness of the outer and inner side walls, respectively. Parameter $V_{1}$ works on the depth and $R_{1}$ works on the width of the wells. With these standalone functions, it is quite convenient for us to tune the parameters and make the system gain an optimal status. Besides, it is obvious that the WSGP can form a much steeper walls and flatter well bottoms. This seems to make the particle oscillate more easily. In the traditional potential model, the value of potential may be boundless with a position $x$ large enough. In this circumstance, the system output may be divergent with a large input signal or noise which exceeds the threshold. So the SR effect will not occur. But for the proposed model, it has an upper limit of potential value of $V_{1}$. So it is more probable for the output to be convergent and has a wider input amplitude bandwidth. This indicates the SR effect comes up much more easily which will generate a desired output. In our next work, all the description of advantages for new model will be verified by both simulated and experimental results.

3.2. Output Evaluation and Diagnosis Scheme. To optimally detect the weak signal of driving frequency from the background noise, the WSGSR can be conducted by adjusting the WSGP parameters until the optimal SR output signal which we get with the method mentioned above is achieved. While this is not enough, we need a target to evaluate the performance of SR effect. Here, we employ the output SNR as a criterion to assess the result with which we can judge the optimal condition of our SR system. The output SNR is defined as the power spectral density of the driving signal divided by the average background noise in a small frequency bin around the driving frequency [9]. In order to have a simple computational process, we simplify the definition and expression of SNR into the form $[6,8]$

$$
\mathrm{SNR}=10 \log _{10} \frac{A_{d}}{A_{n}},
$$

where $A_{d}$ and $A_{n}$ are the amplitude values corresponding to the driving frequency $f_{d}$ and the strongest interference frequency $f_{n}$ (which means the frequency with the largest amplitude except the driving frequency) in the power spectrum, respectively. A higher SNR implies a better discrimination between the periodic signal and the noise. The proposed model and the signal processing also aim at gaining a higher SNR.

The SR model is shown as in (4). In the signal processing, it mainly refers to the numerical analysis. In practical application, the periodic signal and noise are all definite with a known potential model. So we need to solve this differential 
equation to gain its output $x(t)$. Equation (4) is a typical first-order differential equation, so the discrete fourth-order Runge-Kutta method [16] is used here. With this method, we can get the output easily.

Then based on the new WSGSR model, we propose a new scheme of bearing fault diagnosis with this SR system, which is presented in Figure 4. In practical applications, the vibration or acoustic signal is usually acquired by accelerometers or microphones attached from bearings which is always modulated to a high frequency band. So in the proposed scheme, the envelope of the analyzed signal is firstly achieved based on the Hilbert transform (HT). Secondly, the modified signal is sent to the WSGSR system in (7) with presetting parameters. In next step, we calculate the output SNR of each set of parameters in the parameter searching space which is used to obtain the optimal parameters corresponding to the maximal SNR. Substitute the optimal parameters to the model and calculate the finally output, then go ahead with the spectral analysis in the spectrum of the system output by Fourier transform, and realize the fault frequency identification.

\section{System Performance with Simulated Signal}

4.1. Intuitional Output of WSGSR. To have a general presentation for the effect of the proposed SR model, we simulate a sinusoidal signal with frequency of $f_{d}$ and amplitude of $A$ surrounded with Gaussian white noise of intensity $\sqrt{2 D}$

$$
s(k)=A \sin \left(2 \pi \frac{f_{d}}{f_{s}} k\right)+\sqrt{2 D} \xi\left(\frac{k}{f_{s}}\right) .
$$

Firstly, we take $A=0.5$ and $f_{d}=100 \mathrm{~Hz}$. The sampling frequency $f_{s}$ is taken as $20 \mathrm{kHz}$ here. Figures 5(a) and 5(b) are the sinusoidal signal and the mixed signal with noise intensity of 1.5 in time domain, respectively. In Figure 5(b) we can hardly find the periodic component. Figure 5(c) is the potential model of traditional bistable SR as in (5) while Figure 5(d) is the potential model of WSGSR as in (3). All the initial parameters are set as shown in the figures to guarantee a similar potential feature between the two models. Both the barrier heights are 0.25 and the balance positions are set on $x= \pm 1$ which indicate the distance between the two potentials. The similar features can offer us the fairness in comparison of the ability and advantages between the different models. Since we have both the input signal and the SR model as in (6) or (7), we will try the numerical analysis next.

As the power spectral density of SR output meets the Lorentzian distribution which is characterized by concentrating most of noise energy into the low frequency region. So there is a small parameter limitation for SR effect [2]. In practical signal processing, the sampled signal parameters such as the frequency and amplitude of a periodic signal usually exceed the limitation of small parameter SR. In order to normalize the frequency of the original signal to be a small one, the rescaling frequency method is employed here [3]. We take the rescaling ratio $R=1000$ which changes the characteristic frequency from $100 \mathrm{~Hz}$ to $0.1 \mathrm{~Hz}$ that is smaller than one. With a noise intensity of 1.5 and 8192 points data length (all the numerical analysis in this paper is under the same condition), Figures 5(e) and 5(f) indicate the outputs of the two different models, respectively. We can find that, under the same condition, the WSGSR system makes the particle transit between the two potential wells more easily than the traditional system. We have given the explanation before as the potential walls are steeper and this causes the particle to be rebounded rapidly gaining a higher speed. With this advantage, the transition between the two wells comes up much more easily in the proposed SR system which will make the SR effect more likely to be available. Then, keeping all the parameters except the noise intensity, we increase it to 3 and recover the output as Figures $5(\mathrm{~g})$ and $5(\mathrm{~h})$. As a result, the particle oscillates between the potential wells more severely and the transition comes up more obviously. This fits the SR effect well and the transition in Figure 5(h) is more coherent. It profits from the fact that the potential wells in WSGSR are flatter so when the particle locates in one of them, it moves unobstructed. Further on, we reduce the characteristic frequency to $50 \mathrm{~Hz}$ while keeping the same rescaling ratio and the rescaling frequency will be $0.05 \mathrm{~Hz}$. In this condition, the two systems' outputs are shown as in Figures 5(i) and 5(j). As expected, we get the output more approximate to the original signal with its waveform highly restored. We still can find a better result of WSGSR which has 15 peaks in 0.3 seconds distinctly. Next step, we try to find the optimal parameters for a certain simulated bearing fault signal that is periodic unilateral attenuation impulse and reveal the proposed model's deeper performance.

4.2. Optimal Output with Simulated Bearing Fault Signal. In practical bearing fault diagnosis, bearing fault signal is always present in the form of impactive series with a fault frequency modulated to a high level [24]. So a periodic unilateral attenuation impulse discrete signal with Gaussian white noise is selected as the simulated bearing fault signal according to the equation

$$
\begin{aligned}
s(k)= & A \sin \left(2 \pi \frac{f_{m}}{f_{s}} k\right) \cdot \exp \left(-d \cdot \bmod \left(f_{0} k, f_{s}\right)\right) \\
& +\sqrt{2 D} \xi\left(\frac{k}{f_{s}}\right) .
\end{aligned}
$$

Here $\bmod (a, b)$ is the remainder of $a$ divided by $b$ which controls the impulses that appear periodically. $A=1$ means the amplitude and $f_{m}=1200 \mathrm{~Hz}$ indicates the modulation frequency of modulating signal. $f_{s}=20 \mathrm{kHz}$ means the sampling frequency. $d=600$ reflects the attenuation rate and we take the $f_{0}=100 \mathrm{~Hz}$ here as the fault frequency or driving frequency. $\xi(t)$ is the noise with zero mean and a unit variance with noise intensity $\sqrt{2 D}=1.8$. We take a simulation with 8192 points which means the $k$ changes from 1 to 8192 . The generated signals without and with the noise are shown as two figures in Figure 6(a), respectively. Figure 6(b) shows its envelope signal and power spectrum. As a modulated signal, we need to have it demodulated and we use HT method referred to in 3.2 (note that the envelope signals in the following cases are all processed in the same way). Before the demodulation process, filtering of noises 


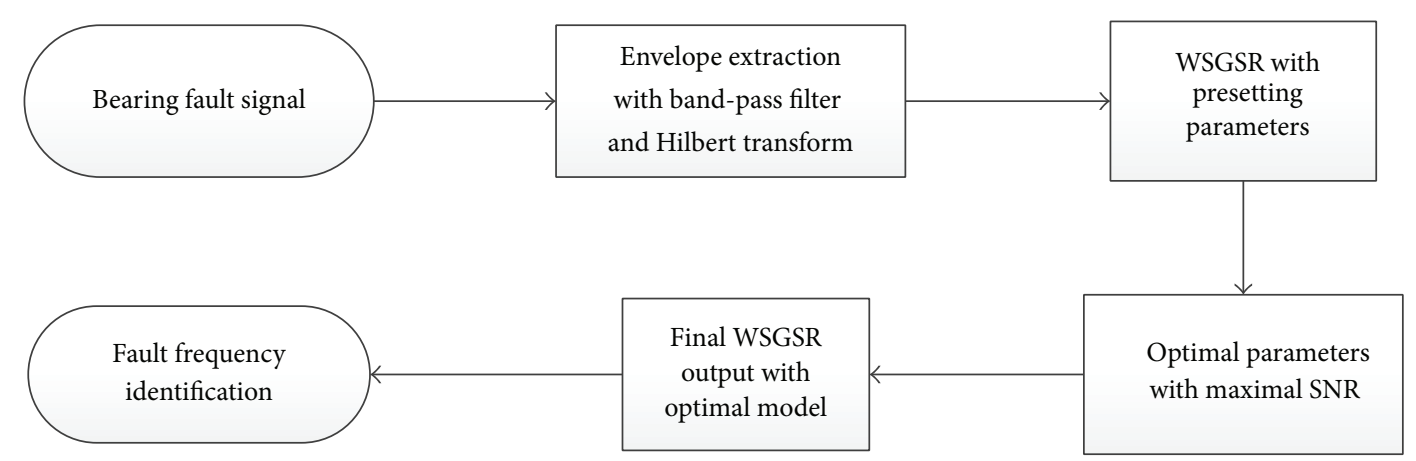

FIGURE 4: Proposed scheme of bearing fault diagnosis with WSGSR.

that are located in the uncorrelated bandwidth is taken. We can find the signal's main energy band from the spectrum directly and here a four-order Butterworth band-pass filter with bandwidth of $1200 \mathrm{~Hz}$ centered at $1200 \mathrm{~Hz}$ is used. We can find that the fault frequency $f_{0}$ is extracted successfully with HT and band-pass filtering while interferences around $f_{0}$ can be seen clearly which makes it hard to identify.

Sending the above signal demodulated by HT into the traditional bistable SR model, we get the output signal and its power spectrum as in Figure 6(c). The parameters are changed to $a=1$ and $b=16$ to guarantee an easy appearance of SR which might not be the optimal ones with rescaling ratio of 1000. Pay attention to that the potential barrier height is now $a^{2} / 4 b=1 / 64$ with equilibrium positions of $\pm \sqrt{a / b}=$ \pm 0.25 . From the output we can find the component of $f_{0}$ is highlighted in the spectrum while the noise components are still frustrating to some extent. What is more is that the output signal in time domain is not satisfying which means the particle cannot transmit between the wells smoothly. Maybe it can be solved by the optimization of parameters. Later we take the WSGSR model with the similar features and the parameters are fixed to match the features of traditional one, where $V_{1}=3, R_{1}=0.5, a=0.4, V_{2}=1 / 64$, and $R_{2}=0.4$. We can calculate the two models have the same barrier height and equilibrium positions. The output of the proposed model with the same input signal is shown in Figure 6(d) as well as its spectrum. It can be seen from the power spectrum that the amplitude of component $f_{0}$ is higher than that of the other noise components which makes it easily recognized. Besides, the components of lower frequency are weaker than that of the output in traditional SR. So we can find the signal of WSGSR in time domain more ideal which is to say the particles in traditional one move harder as a result of too many low frequency components. It indicates that the particles in new SR system transmit more easily between the two potential wells with the same barrier height and equilibrium position. As the differences between the two models are shape of walls and bottoms, this may owe to the steeper walls and flatter well bottoms which we have described before. The results also show us the superiority of WSGSR over the traditional SR system.

The outputs we get in Figure 6 are not the optimal results as the parameters are preset. So we need to find out a set of parameters to gain the highest SNR as in (8) and an optimal output. Here SNR acts as the criterion. We can take an iterative algorithm to find each optimal parameter within a certain range. After this work we can get the optimal system to match the input signal. The specific steps are introduced as below.

(1) Before we search for the optimal output, we need to have the signal preprocessed. As the original signal is modulated, we firstly need to have it band-pass filtered. Then use Hilbert transform to process the signal. The output will be demodulated successfully. After this, we can send the signal into WSGSR system of different parameters to gain the output.

(2) Calculate the power spectrum of the output waveform and obtain the SNR. Search the maximal SNR in the parameter space that is constructed by the varying variables $V_{1}, R_{1}, a, V_{2}$, and $R_{2}$ in certain ranges and then obtain the optimal parameters corresponding to the maximal SNR. If all the optimal parameters are not on the boundaries of the search ranges, we have found the optimal result successfully or else extend the range and try the searching step again.

(3) Finally, substitute the optimal parameters (as we get in Figure $7 V_{1}=9, R_{1}=0.6, a=0.5, V_{2}=$ 0.15 , and $R_{2}=0.9$ ) and get the output waveform as the detected weak signal. Further calculate the corresponding power spectrum for identification of the driving frequency and make a diagnostic conclusion. Figure 7 shows us the finally optimal output waveform and its spectrum with a clearly highlighted fault frequency or driving frequency of $100 \mathrm{~Hz}$. When comparing it to the initial output as in Figure 6(d), we can find less noise around the driving frequency and a more distinct result which verifies the effectiveness of the optimization process.

4.3. Performance Analysis and Comparison. In the optimization process above, we mentioned that optimal parameters can be obtained according to the maximal SNR in a certain range. It means that there will appear a peak for SNR with only one of the parameters changing. With a decided input signal, the system output only depends on five potential parameters. We fixed four of them and varied the other one of the WSG potential in turn and then calculated the SNR of the output signal via (7) with input signal same as last section 


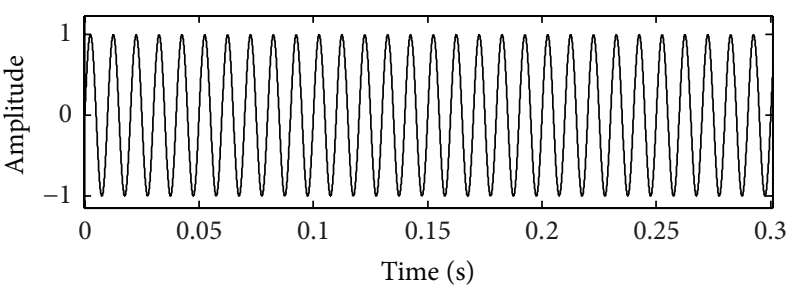

(a)

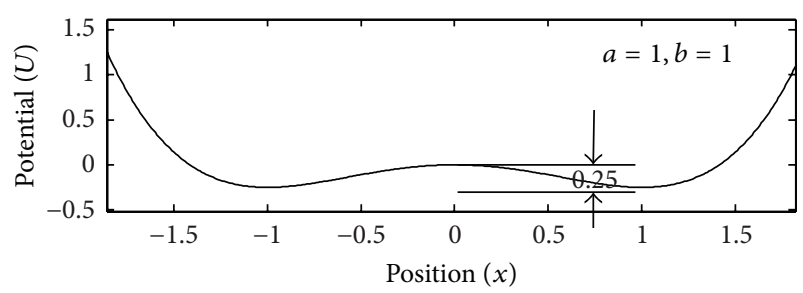

(c)

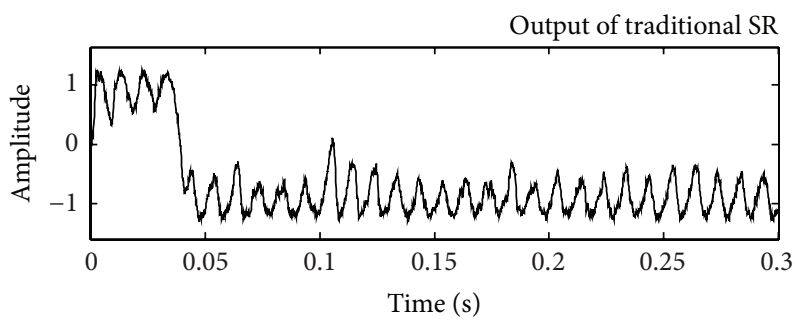

(e)

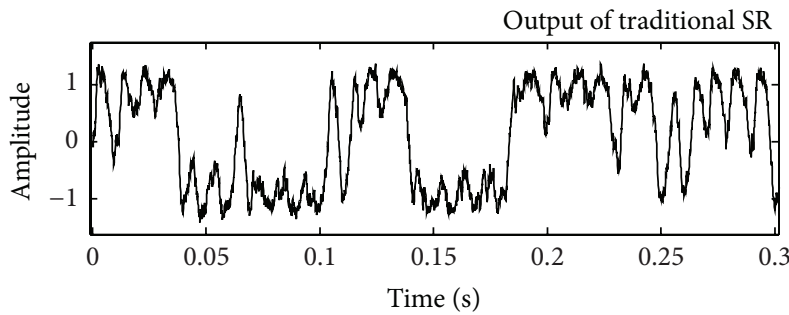

(g)

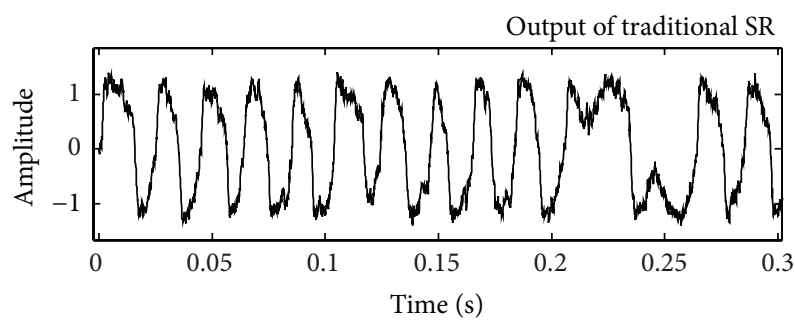

(i)

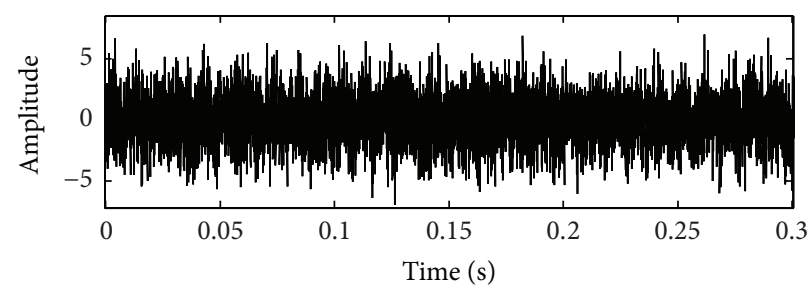

(b)

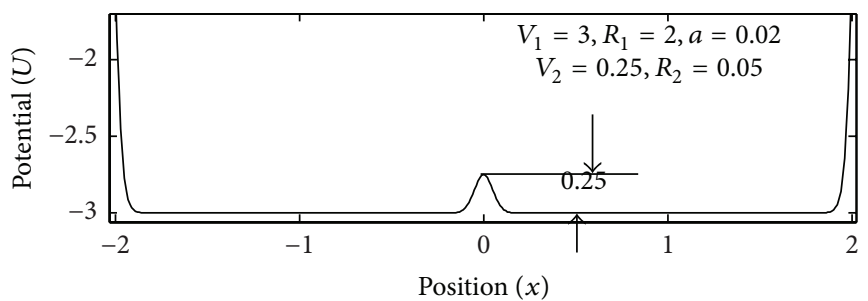

(d)

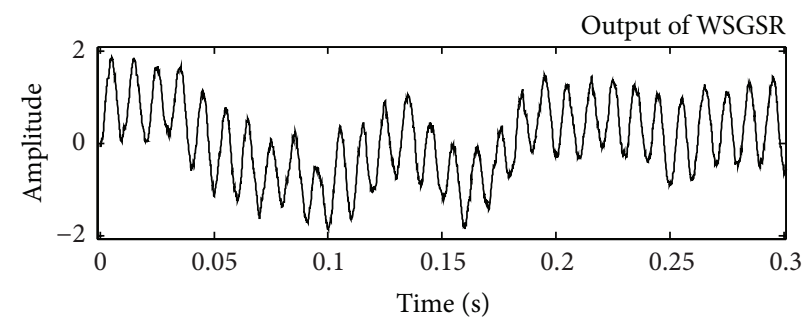

(f)

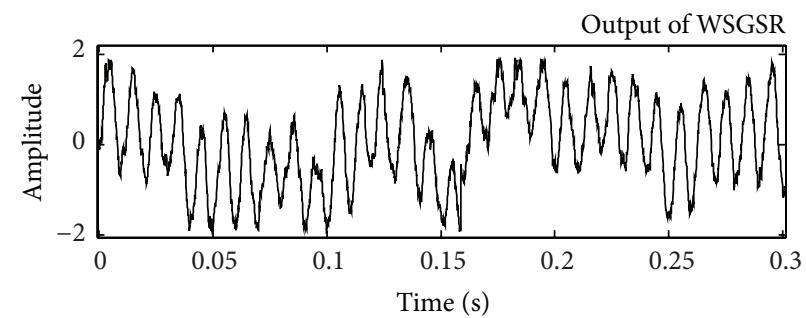

(h)

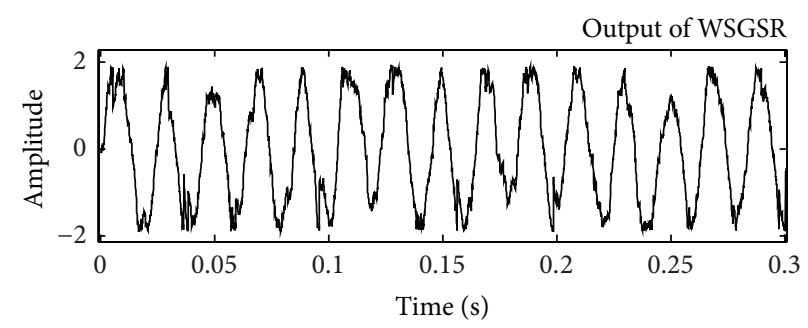

(j)

FIGURE 5: Outputs signal of different SR models with a sinusoidal signal mixed with noise: (a) sinusoidal signal, (b) original signal mixed with noise, (c) traditional bistable potential with $a=1, b=1$, (d) WSGP with $V_{1}=3, R_{1}=2, a=0.02, V_{2}=0.25$, and $R_{2}=0.05$, (e) and (f) outputs of the two different models with noise intensity of 1.5 and driving frequency of $100 \mathrm{~Hz},(\mathrm{~g})$ and (h) outputs of the two different models with noise intensity of 3 and driving frequency of $100 \mathrm{~Hz}$, and (i) and (j) outputs of the two different models with noise intensity of 1.5 and driving frequency of $50 \mathrm{~Hz}$.

whose driving frequency is $100 \mathrm{~Hz}$ and noise intensity of 1.8 . Figure 8 shows us the SNR variation trend with the changing of five parameters. The thick red curve is the smoothing fitted curve (via piecewise polynomial interpolation method) of the original SNR curve (thin one). In every figure, we vary sequentially each one of the parameters while keeping the others fixed. For example, in Figure $8(\mathrm{a})$, we make $R_{1}=0.5$, $a=0.2, V_{2}=0.3$, and $R_{2}=0.8$ while varying $V_{1} \in[0.05,10]$. The results offer us the hints that the proposed model has a similar feature as traditional SR system in which the SNR 

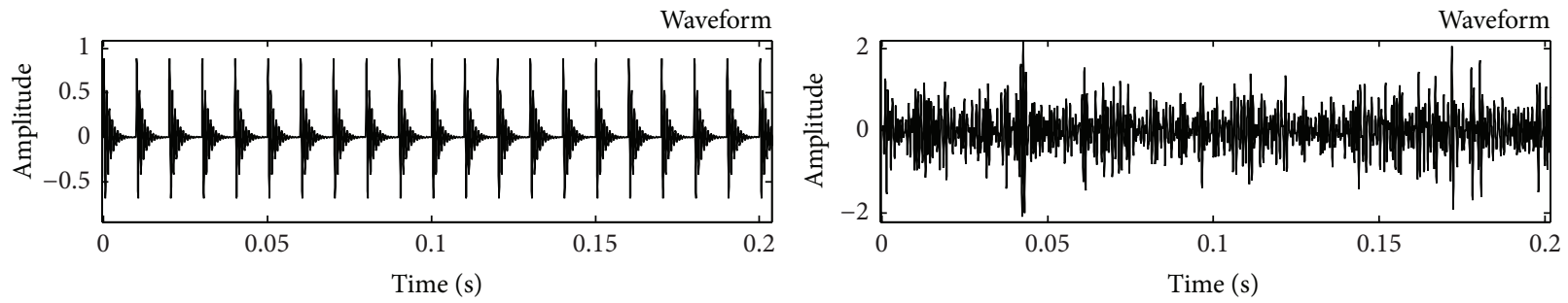

(a)
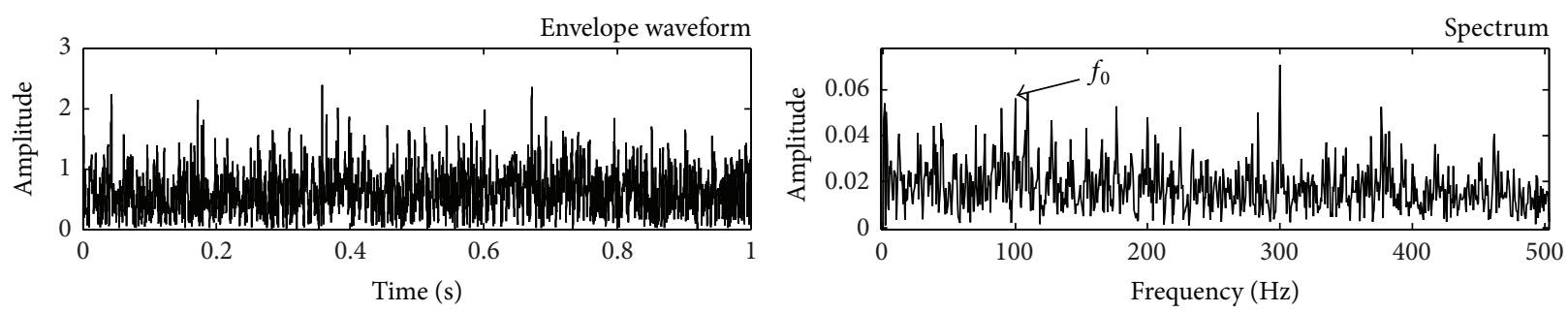

(b)
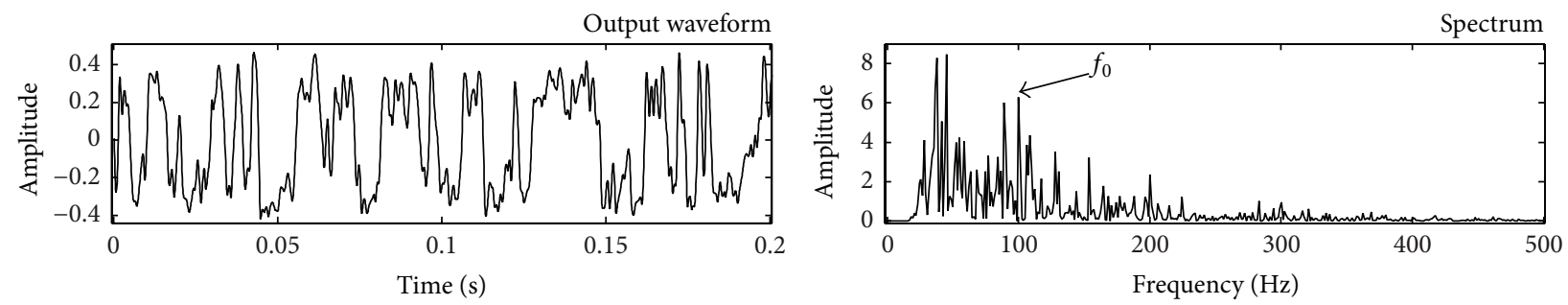

(c)
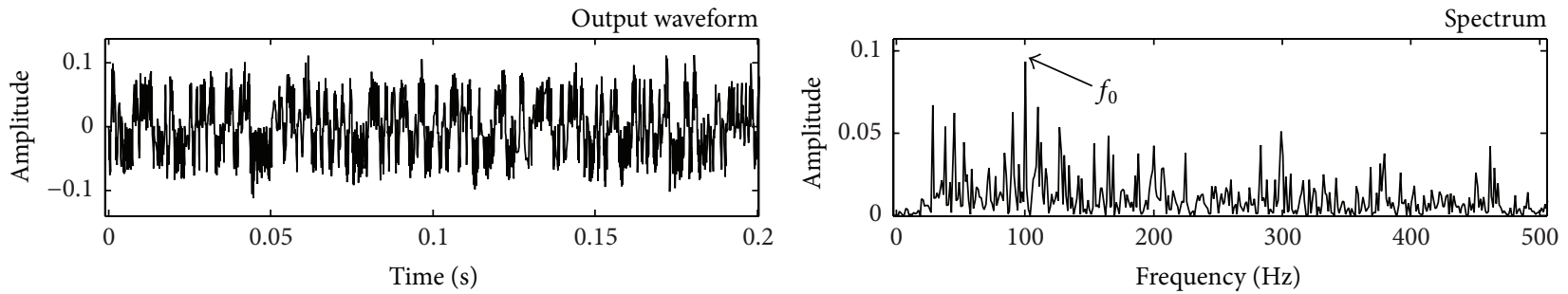

(d)

FiguRE 6: Simulated bearing fault signal and outputs of different models: (a) periodic unilateral attenuation impulse signal of $100 \mathrm{~Hz}$ and mixed signal with noise intensity of 1.8, (b) envelope signal and power spectrum filtered with $[600 \mathrm{~Hz}, 1800 \mathrm{~Hz}]$, (c) output of traditional bistable SR model and its power spectrum with $a=1, b=16$, and (d) output of WSGSR model and its power spectrum with $V_{1}=3, R_{1}=0.5$, $a=0.4, V_{2}=1 / 64$, and $R_{2}=0.4$.
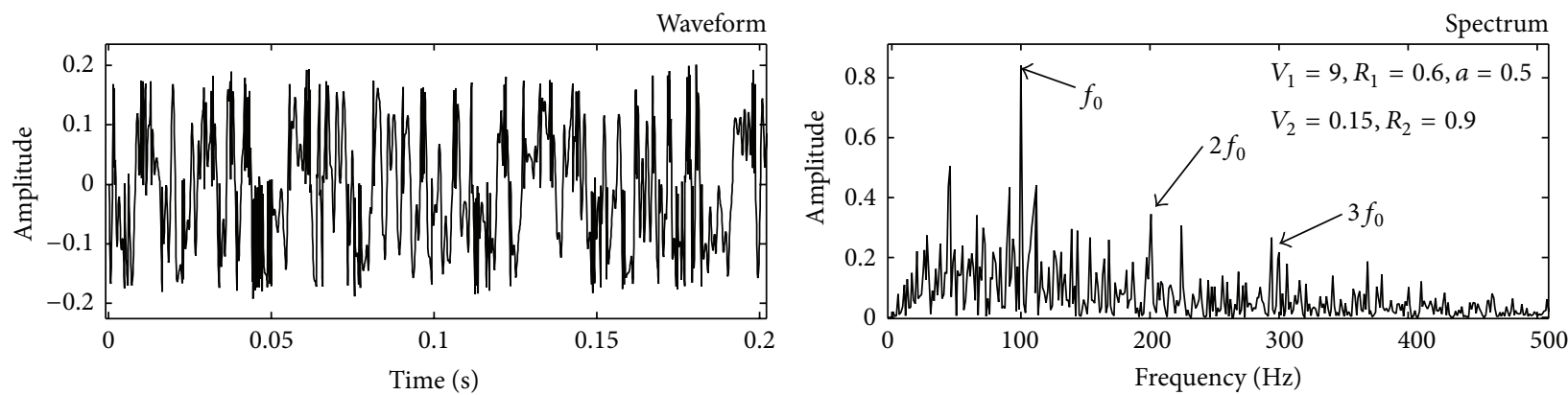

Figure 7: Optimal result for Figure 6(d) and its spectrum with $V_{1}=9, R_{1}=0.6, a=0.5, V_{2}=0.15$, and $R_{2}=0.9$. 


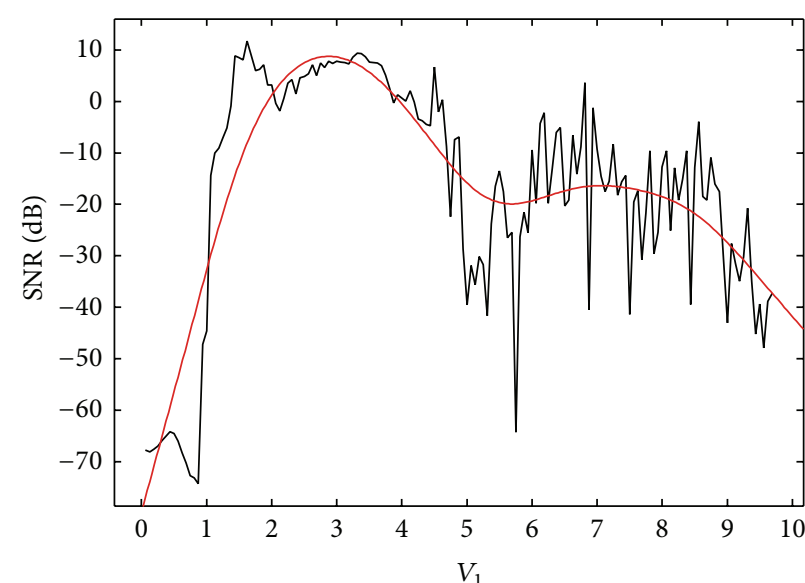

(a)

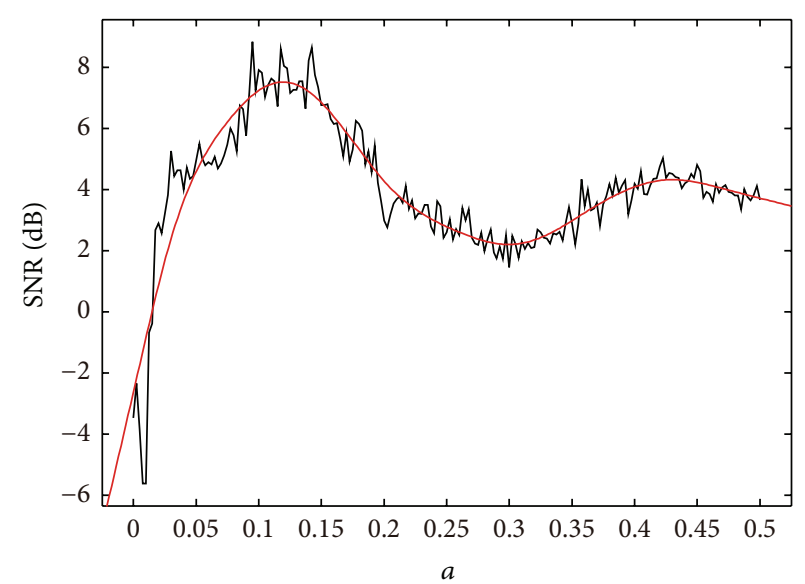

(c)

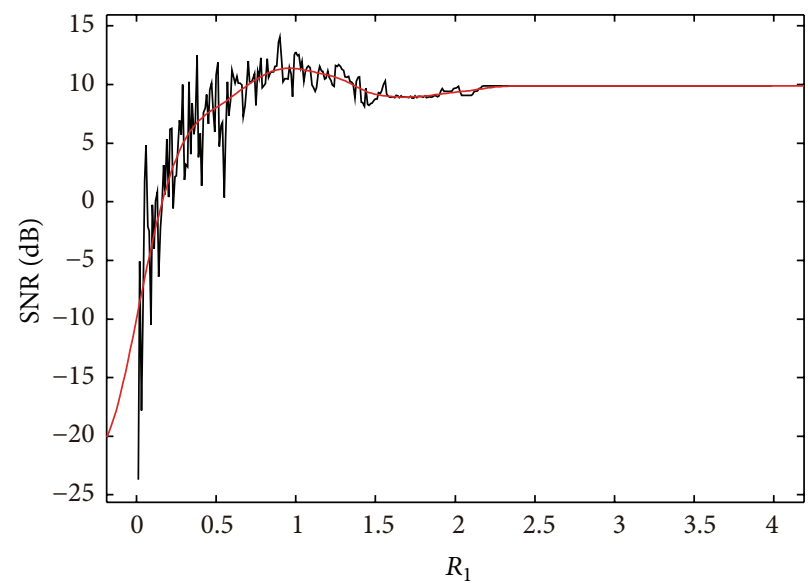

(b)

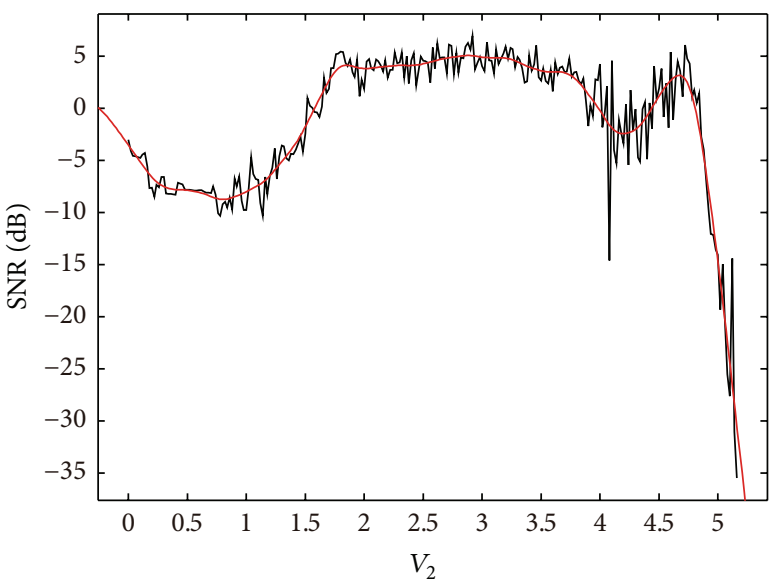

(d)

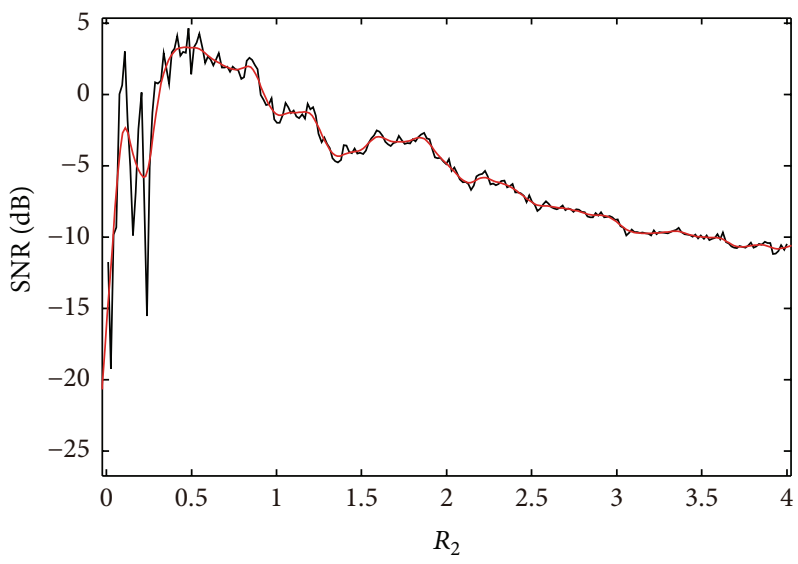

(e)

FIGURE 8: SNRs variation trend with the changing of five parameters in different ranges: (a) $[0.05,10],(b)[0.01,4],(c)[0.1,0.5],(d)[0,5.5]$, and $(\mathrm{e})[0.01,4]$.

variation tendency firstly increases with the increasing of the variable reaches a maximum and then decreases when the variable keeps increasing. Figures 8(d) and 8(e) especially offer us the evidence that the proposed method can improve the output SNR of WSSR, where $V_{2}=0$ further. Because, in our method, even if we fix $V_{2}=0$ firstly, we can get an optimal output of WSSR model. Then we can tune the parameters $V_{2}$ and $R_{2}$ to get a higher SNR and a better output signal via these two figures. That is to say, an optimal output with the highest SNR of WSGSR can be obtained by parameter tuning. Besides, with the increasing of $V_{2}$ from 0 , we find that the SNR decreases firstly. Later, with the changing of $V_{2}$ further, the 
output SNR begins to increase and even exceeds the situation under $V_{2}=0$. This can be explained easily. Then the adding of $V_{2}$ in the beginning plays a negative role to the output. But with the changing of its value, when the system comes to a best matching, the barrier will serve a positive function. It means that the employment of $V_{2}$ does not always have benefit unless it is under an optimal situation.

Following on, we make a new simulated signal with a higher driving frequency of $f_{0}=200 \mathrm{~Hz}$ whose amplitude is fixed at $A=0.8$ and a noise intensity of $\sqrt{2 D}=1.5$ in (10). In Figure 9(a), it stands for the original waveform and its power spectrum. All the other parameters are the same as in Section 4.2. From its spectrum in Figure 9(a), we can easily get the signal's main energy band and design the band-pass filter. Figure 9(b) shows the envelope signal generated by HT and its spectrum after a filter with bandwidth of $1600 \mathrm{~Hz}$ centered at $1200 \mathrm{~Hz}$. Without any process except for the filtering and HT, the driving frequency can be hardly identified. Sending the envelope signal into the traditional bistable SR model, optimizing the parameters $a$ and $b$, we can get a best combination of parameters of 0.2 and 46 , respectively, with the rescaling ratio $R=1000$. Its output is shown as in Figure 9(c) with SNR of $2.89 \mathrm{~dB}$. We find that the driving frequency at $200 \mathrm{~Hz}$ is well extracted while there are still too many noises of low frequency that obstruct our judgment. Later, the proposed WSGSR method is utilized to deal with the filtered envelope signal and after parameters tuning we get the optimal values for parameters: $V_{1}=14, R_{1}=0.2, a=0.4$, $V_{2}=0.7$, and $R_{2}=0.5$. The optimal output in Figure 9(e) shows us a distinct characteristic frequency with noise highly weakened and the maximal SNR reaches $4.71 \mathrm{~dB}$. Specially, we set $V_{2}=0$ when the model becomes WSSR; we get the optimal parameters combination of $V_{1}=8, R_{1}=0.45$, and $a=0.7$ and its output is shown in Figure 9(d). It is also a satisfying result with SNR of $4.02 \mathrm{~dB}$. Compared with the result of traditional model, the WSGSR output can keep the original waveform better and offer a larger enhancement of the weak impulse signal. Besides, the differences between Figures 9(d) and 9(e) show us the probability that the proposed model may have a better performance which contains the situation of WSSR and can reach a higher output SNR. The amplitude of the driving frequency in its output gets 4.008 while it is only 0.524 when $V_{2}=0$. This means higher output energy with the help of potential barrier like in a traditional bistable SR with amplitude of 1.791 although the SNR is the lowest.

In order to verify our conjecture about the proposed method, we make a simulation to compare the capacity of different models when dealing with signal under different noise intensity or driving frequency.

Firstly, fixing the frequency at $200 \mathrm{~Hz}$ still, we set the amplitude of attenuation impulse signal in (10) $A=0.8$ and the noise intensity $\sqrt{2 D}$ varies from 1.1 to 2.3 with step of 0.2 with data length of 10000 points. Then under interference of each noise intensity scale, we can gain every optimal output with a maximal SNR. The output SNR is shown as in Figure 10(a) with the changing of noise intensity. The three different results are yielded by the traditional bistable SR method, the WSGSR method with $V_{2}=0$ (WSSR), and the proposed method, respectively. What is obvious is that the three curves all indicate a decrease of SNR with the increase of noise intensity. This confirms our cognition well. But there is several other information which the figure offers us. We can find a higher SNR with the proposed method than the traditional SR and the employment of $V_{2}$ which improves the performance of WSSR offers us a better result. When treating the WSSR as a special case of WSGSR, the proposed model shows the superiority in detecting weak impulse signals that are submerged in noise of different level. Besides, when comparing the distinction between them, we find an increasing trend (from $0.19 \mathrm{~dB}$ to $0.81 \mathrm{~dB}$ ) with the increasing noise intensity which means the heavier noise, the more superior performance the proposed model has relatively.

Secondly, to observe the performances of different models under different driving frequency, we make another series signal. In them, the amplitude of attenuation impulse signal is set as $A=0.8$ with noise intensity of 1.5 . They have varying driving frequency from $50 \mathrm{~Hz}$ to $1000 \mathrm{~Hz}$ with 6 points. To research the effectiveness and bearing capacity to different driving frequency, we still calculate the optimal output of signal under each driving frequency and get the output SNR. Making a permanent rescaling ratio of 1000 , the three curves in Figure 10(b) show us the results. All the three SNRs increase firstly because the higher driving frequency, the higher energy it will contain under same amplitude as (10) gives. We can find a more stable performance with our proposed model than the traditional bistable SR with the changing of driving frequency and the parameter $V_{2}$ still works. Although the larger frequency impacts the output SNR, it is not as serious as the traditional bistable SR. It coincides with the result in Section 4.1 well. It is obvious that as a restriction of small parameters, the equivalent driving frequency of traditional bistable SR changes from 0.05 to 1 with rescaling ratio of 1000 which gets farther and farther from the condition. So as a result, with a frequency large enough, the output SNR begins to decrease rapidly. In our proposed model, the SNR also decreases after a certain frequency but not as severe as it. This means the proposed model has an advantage when dealing with high frequency signal over the traditional one.

Based on the above analysis, our conjecture is verified. With the proposed WSGSR model, signal extract under heavy noise or bearing fault diagnosis can be obtained by tuning the independent parameters and it is proved to have a better adaptability in detecting the weak signal with different noise intensities and different driving frequencies. After these simulation works, the model will be applied in experimental bearing fault signal to value its engineering application.

\section{Engineering Application}

To verify the effectiveness and efficiency of the proposed method in engineering applications, a set of train bearing signals carrying fault information are analyzed according to the scheme of bearing fault diagnosis with proposed WSGSR model in Figure 4. The bearing outer-race defective and inner-race defective datum are generated from a rolling bearing used separately. 

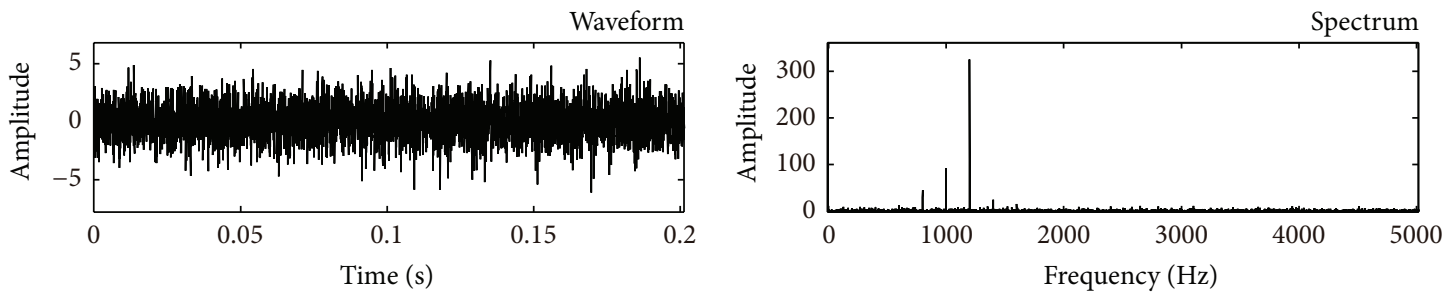

(a)
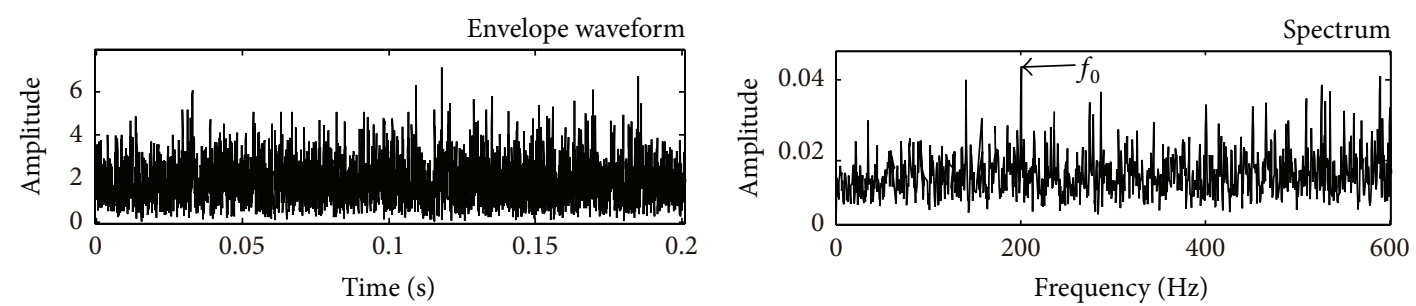

(b)
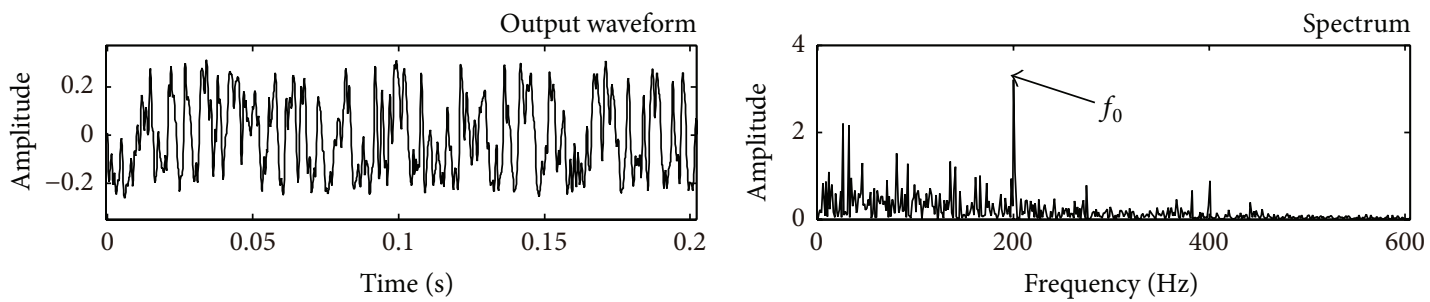

(c)
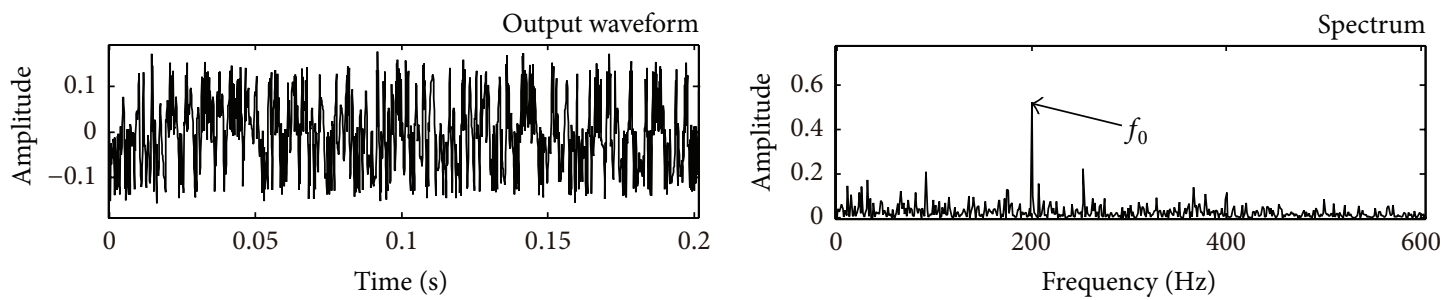

(d)
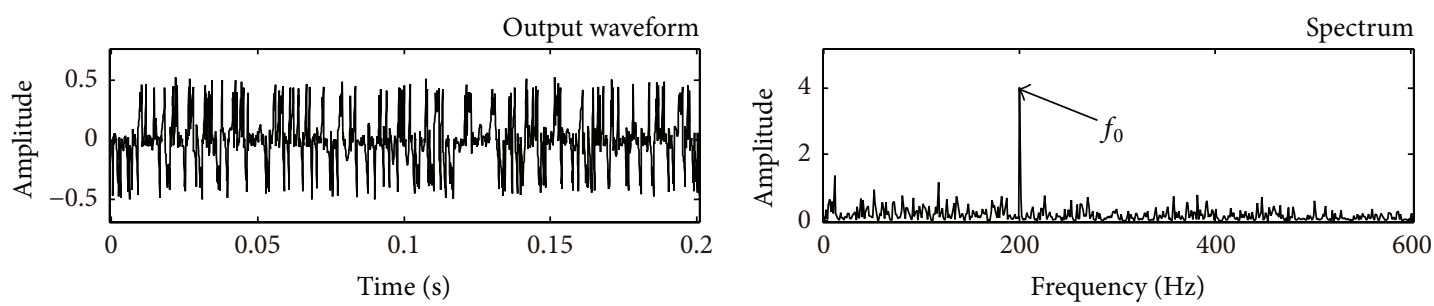

(e)

FIGURE 9: Outputs of different models with driving frequency of $200 \mathrm{~Hz}$ : (a) original waveform and its power spectrum with $A=0.8, \sqrt{2 D}=$ 1.5 , (b) envelope signal and its spectrum filtered with $[800 \mathrm{~Hz}$ and $1200 \mathrm{~Hz}]$, (c) output of bistable SR model and its spectrum with $a=0.2$, $b=46$, (d) output of WSGSR model and its power spectrum with $V_{1}=8, R_{1}=0.45, a=0.7$, and $V_{2}=0$ (WSSR), and (e) output of WSGSR model and its power spectrum with $V_{1}=14, R_{1}=0.2, a=0.4, V_{2}=0.7$, and $R_{2}=0.5$.

TABLE 1: Specification of the train bearing NJ(P)3226X1.

\begin{tabular}{lccccc}
\hline Type & $\begin{array}{c}\text { Diameter of the } \\
\text { outer race }\end{array}$ & $\begin{array}{c}\text { Diameter of the } \\
\text { inner race }\end{array}$ & $\begin{array}{c}\text { Pitch diameter } \\
(D)\end{array}$ & $\begin{array}{c}\text { Diameter of the } \\
\text { roller }(d)\end{array}$ & $\begin{array}{c}\text { Number of the } \\
\text { roller }(z)\end{array}$ \\
\hline $\mathrm{NJ}(\mathrm{P}) 3226 \mathrm{X} 1$ & $250 \mathrm{~mm}$ & $130 \mathrm{~mm}$ & $190 \mathrm{~mm}$ & $32 \mathrm{~mm}$ & 14 \\
\hline
\end{tabular}




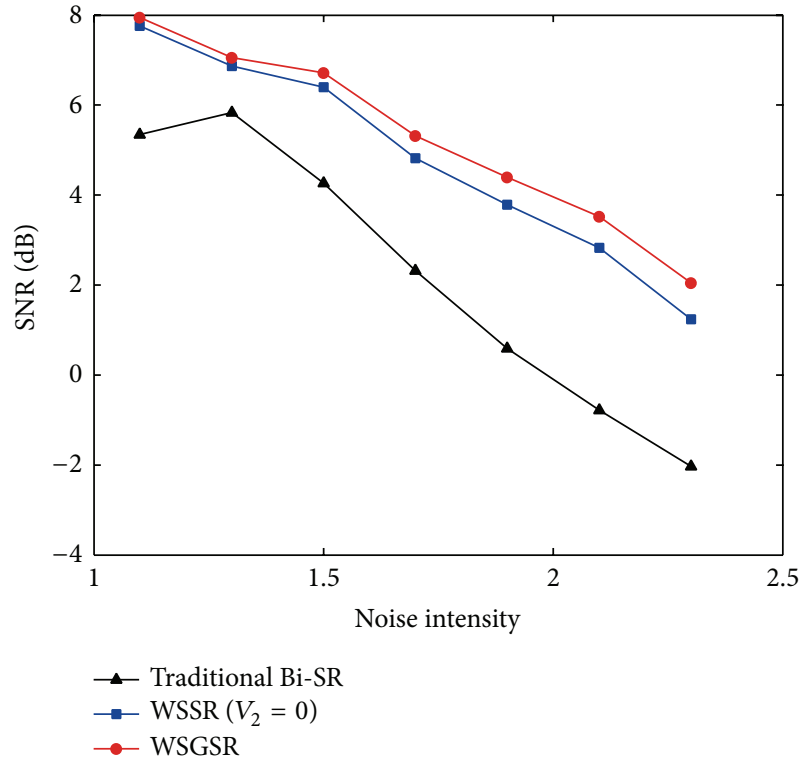

(a)

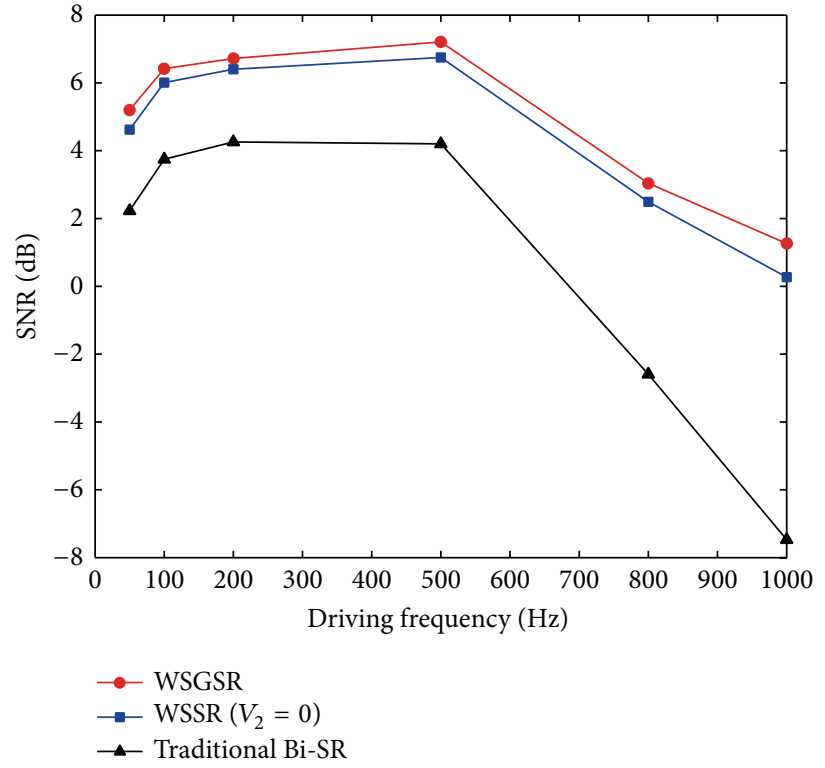

(b)

FIGURE 10: Output SNRs under different noise and driving frequency levels: (a) output SNR with the changing of noise intensity and (b) output SNR with the changing of driving frequency.

The train bearings used in this experiment are single row radial short cylindrical roller bearings, with the type of $\mathrm{NJ}(\mathrm{P}) 3226 \mathrm{X} 1$ with its detailed specification in Table 1. Firstly, we fabricate the artificial cracks of single line faults on the train bearing using wire-electrode cutting machine with the width of $0.18 \mathrm{~mm}$ on the outer and inner race, respectively, as shown in Figure 11. Then the faulty bearing is placed on an experiment platform in Figure 12. In the experiment, the acoustic bearing fault signal was acquired continuously by a microphone (type: 4944-A, manufactory: B\&K Company) being mounted beside the outer race of the bearing placed at the radial direction with the distance of $150 \mathrm{~mm}$. Except for the microphone, a National Instruments (NI) system (PXI1033) equipped with PXI-4472 data acquisition card was used. According to Figure 12, the bearing could be applied with the mechanical load whose magnitude was measured by the pressure sensor and was displayed on a digital tube and it is driven by a motor, which was controlled by a frequency converter to match the anticipant rotating speed.

With all the introduced equipment, we can get the expecting bearing fault signal. In the experiment, the rotating speed is set at $1430 \mathrm{r} / \mathrm{min}$, with a load of about $3 \mathrm{t}$ and the sampling rate of $50 \mathrm{kHz}$. Through the calculation of bearing fault frequency equation with the parameters in Table 1, the value of the failure frequency with the defect on the outer race is $138.74 \mathrm{~Hz}$ and the value of the inner-race defective frequency is $194.93 \mathrm{~Hz}$. Then after trying the experiment, we acquire the acoustic bearing signal with outer-race or innerrace defect. They are used to test the proposed WSGSR model and in order to have a comparison, the envelope signal and traditional bistable SR are also utilized to process the signal. The analysis results are described as below.
5.1. Results with Outer-Race Fault. The analyzed results of the outer-race defective train bearing signal using different methods are displayed in Figure 13. Figure 13(a) shows us the original signal and its spectrum. As a modulated signal, the structure resonance band of the raw signal is between $500 \mathrm{~Hz}$ and $2500 \mathrm{~Hz}$ where the main energy locates. But the fault signal cannot be found in both the waveform and the corresponding power spectrum. Making the signal band-pass filtered with band of $[500 \mathrm{~Hz}, 2500 \mathrm{~Hz}]$ and demodulated we get the envelope signal with spectrum in Figure 13(b). Surrounded by the conspicuous noise components in both high and low frequency regions, the structural defective frequency $f_{\mathrm{BPFO}}$ can hardly be distinguished at the power spectrum with a SNR of $-2.73 \mathrm{~dB}$. Send the envelope signal into a traditional bistable SR system with parameters of $a=$ $1.2, b=46$ which are optimized for the highest output SNR. The output in Figure 13(c) tells us that the noise components have been suppressed and the $f_{\mathrm{BPFO}}$ has been highlighted in the power spectrum with SNR of $1.58 \mathrm{~dB}$, which implies that part of noise energies has been successfully transferred to the driving frequency. But the results are not satisfiable as there are still too many noises of low frequency. Subsequently, the same signal is analyzed by employing the proposed WSGSR method, and the results are exhibited in Figures 13(d) and 13(e). The first one is the output with $V_{2}=0$ which means a WSSR model with the other parameters optimized $V_{1}=16$, $R_{1}=0.35$, and $a=0.2$. In this case the SNR gets further amplified to $2.98 \mathrm{~dB}$. The second one is the optimal results of proposed method with parameters of $V_{1}=2, R_{1}=0.05$, $a=0.2, V_{2}=0.8$, and $R_{2}=0.25$. In frequency domain the component of fault frequency has been successfully extracted with SNR of $5.11 \mathrm{~dB}$. We can find a relatively 


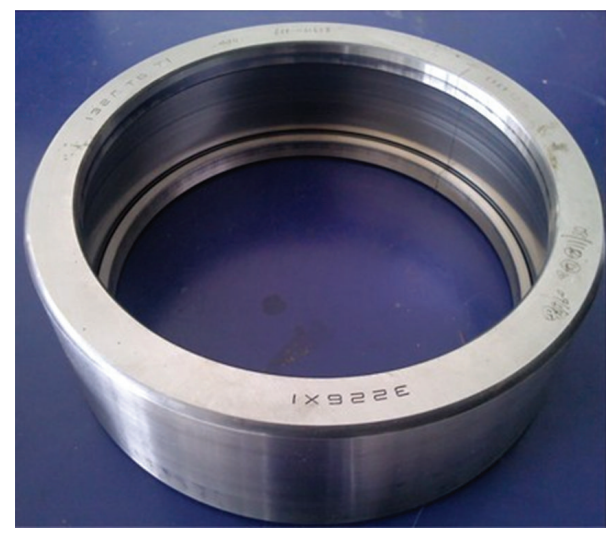

(a)

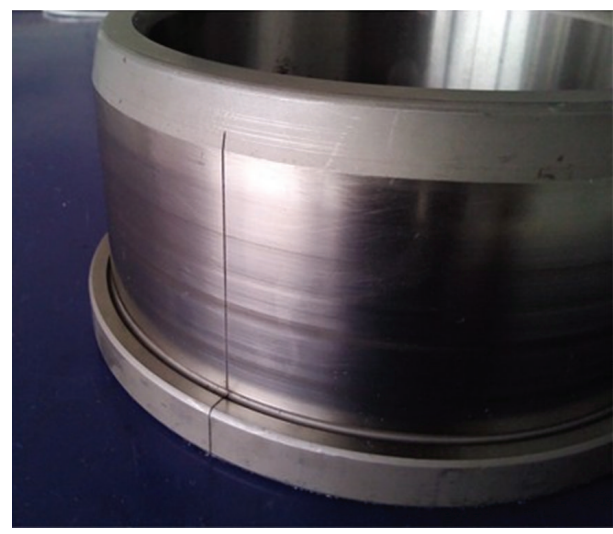

(b)

FIGURE 11: Artificial cracks on the train bearing: (a) outer-race crack and (b) inner-race crack.

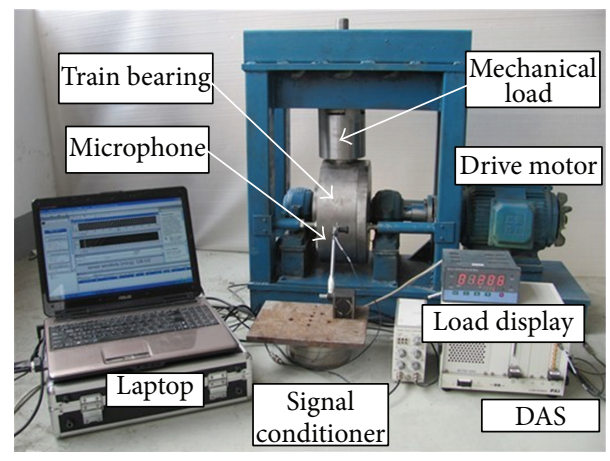

FIGURE 12: Experiment platform for bearing fault signal acquisition.

distinct $f_{\mathrm{BPFO}}$ in the spectrum implying that the proposed method is insensitive to the noise and could instead utilize the background noise to enhance the driving frequency. The differences between results in Figures 13(d) and 13(e) also give evidence of the amelioration compared with WSSR system.

5.2. Results with Inner-Race Fault. The analyzed results of the inner-race defective train bearing signal dealing with different systems are displayed in Figure 14. The original signal and its spectrum are provided in Figure 14(a). With a main energy band from $500 \mathrm{~Hz}$ to $2500 \mathrm{~Hz}$, it delivers no useful information about the fault frequency in both time and frequency domain. Next the envelope signal and its corresponding power spectrum are displayed in Figure 14(b) filtered with band of [ $500 \mathrm{~Hz}$ and $2500 \mathrm{~Hz}$ ]. In the spectrum, we can find a distinct low frequency signed $f_{r}$ with a fairly high energy in the figure which indicates the rotating frequency of $23.8 \mathrm{~Hz}(1430 \mathrm{r} / \mathrm{min})$. With interference of too much bootless frequencies as well as noise, we cannot figure out the fault frequency $f_{\mathrm{BPFI}}$ with SNR of $-3.01 \mathrm{~dB}$ successfully from the envelope spectrum and the defect-induced impulses in time domain are not significant. In the results of traditional bistable SR with $a=0.8$ and $b=38$ which are optimized for the highest output SNR as in Figure 14(c), it gives a better result with SNR $=-0.88 \mathrm{~dB}$. But the interferences are still frustrating as lower frequencies which can still clearly be seen. The results of WSGSR show us a better situation. With fixing $V_{2}=0$ when the model acts as WSSR, Figure 14(d) provides a SNR of $2.92 \mathrm{~dB}$ with optimal parameters of $V_{1}=10, R_{1}=0.5$, and $a=0.1$. But when we add it to 1 with the other parameters $V_{1}=12, R_{1}=1, a=0.2$, and $R_{2}=0.45$ the system gets an optimal condition and the output becomes the one in Figure 14(e). It illustrates in the power spectrum that the $f_{\mathrm{BPFI}}$ has been amplified with a higher power than any other noise components with SNR of $4.76 \mathrm{~dB}$ and we can see the defect-induced impulses in time domain. The results show us the advantages of WSGSR model in bearing fault diagnosis embedded with heavy noises once again.

5.3. Discussion of Experimental Results. With the above subsections, the effectiveness and efficiency of the proposed weak signal detection strategy based on WSGSR have been verified by both the simulated signal and the practical bearing fault signals. The results show a similar verdict.

(1) Considering the two expressions of different SR models as in (6) and (7), it is obvious that the parameters $a$ and $b$ in traditional bistable SR are coupling which means they always work on the potential shape together. With all the parameters affecting potential model's features independently, the coupling effect which the parameters have in a traditional bistable SR model is eliminated well. For example, we can just tune $V_{2}$ to have different barrier height, $R_{1}$ to gain different well width, and a smaller $a$ to make the well wall steeper. As a limitation, the optimal bistable potential that can enhance the periodic weak signal may not be accurately designed with the coupling effect of parameters, so the signal enhancement of the bistable SR is limited. For the WSGP, all the features can be independently designed by their corresponding coefficients. As a result, the WSGP can be adjusted to be matched to different input signal better.

(2) Compared to the shape of traditional bistable potential, the WSGD has a flatter well bottom and steeper well wall. This might make the particle oscillate more easily with less resistance and higher rebound force 

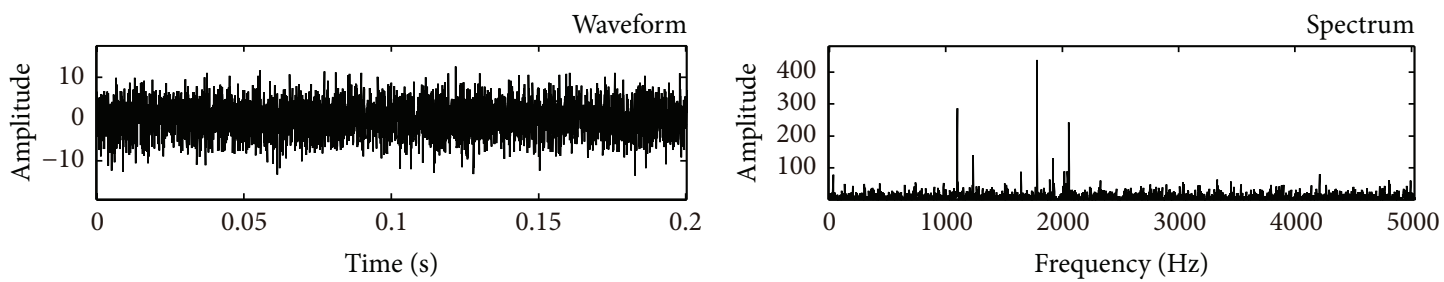

(a)
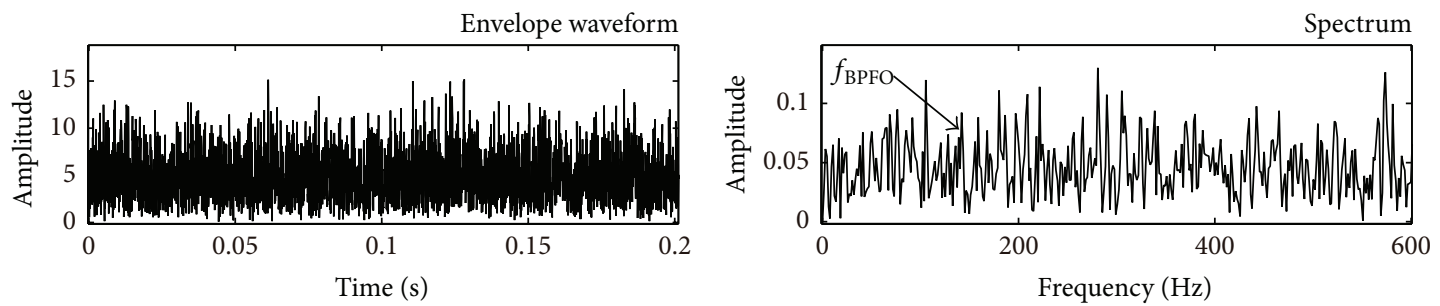

(b)
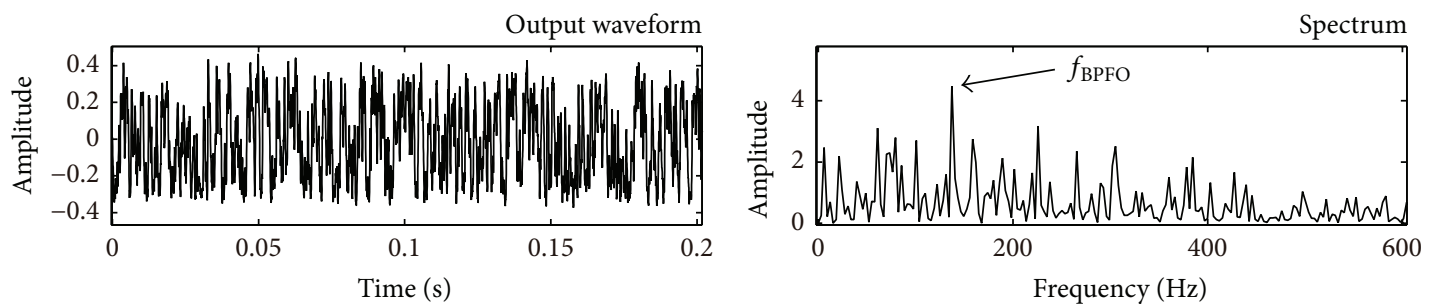

(c)
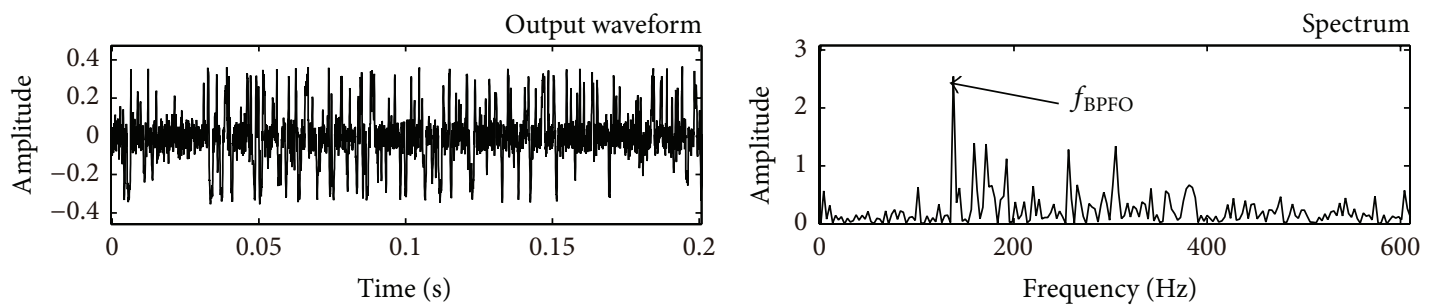

(d)
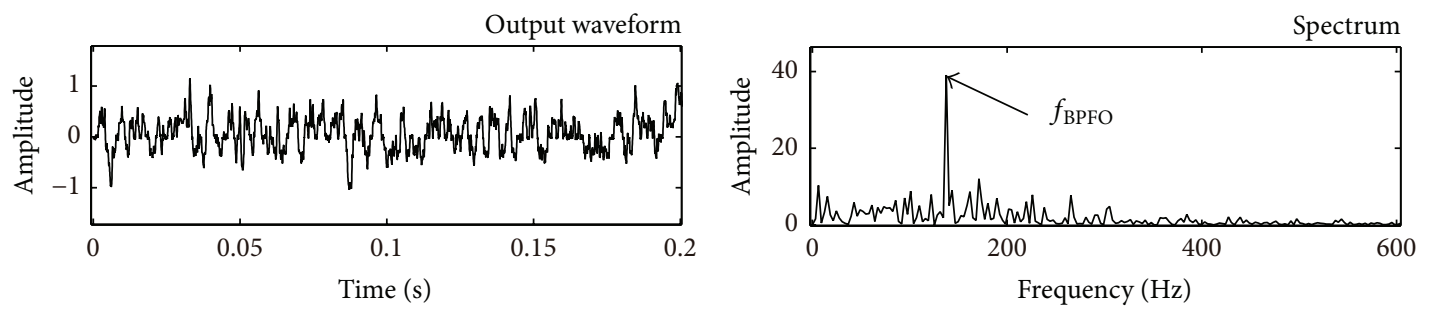

(e)

FIGURE 13: Optimal outputs of different models with outer-race fault signal: (a) original waveform and its power spectrum, (b) envelope signal and its spectrum filtered with [500 Hz and $2500 \mathrm{~Hz}$, (c) output of bistable SR model and its spectrum with $a=1.2$ and $b=46$, (d) output of WSGSR model and its power spectrum with $V_{1}=16, R_{1}=0.35, a=0.2$, and $V_{2}=0$ (WSSR), and (e) output of WSGSR model and its power spectrum with $V_{1}=2, R_{1}=0.05, a=0.2, V_{2}=0.8$, and $R_{2}=0.25$.

which we have described before. The outputs verify our conjecture that the SR effect comes up more easily in the proposed model. The simulating and engineering results all provide the evidence that WSGSR results in a higher output SNR. More commendable, it can work with a wider range of noise intensity and driving frequency. In other words, even with heavier noise and higher frequency, the advantages of proposed method are more obvious and it can offer a better performance than the traditional one. This will owe to the special shape of WSGP.

(3) Besides, as a more general type, the WSGSR not only has the advantages of WSSR which is a particular case but also possesses a better performance. With utilizing of a potential barrier which can be turned 

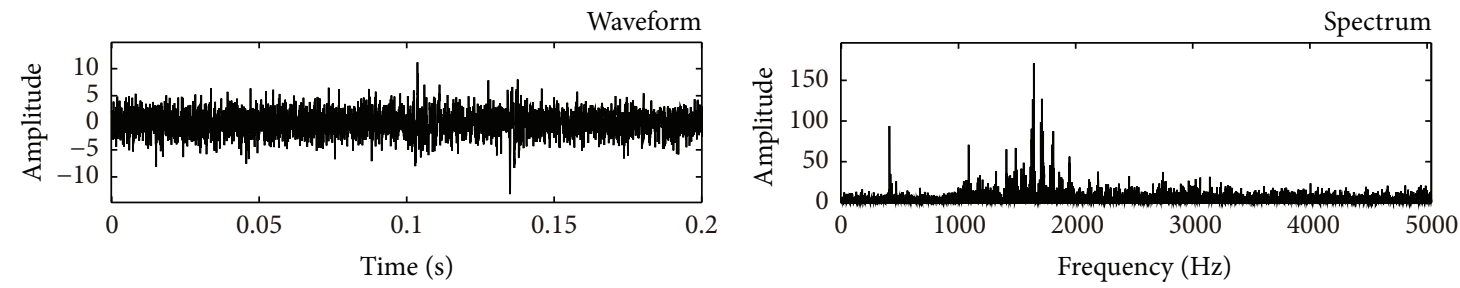

(a)
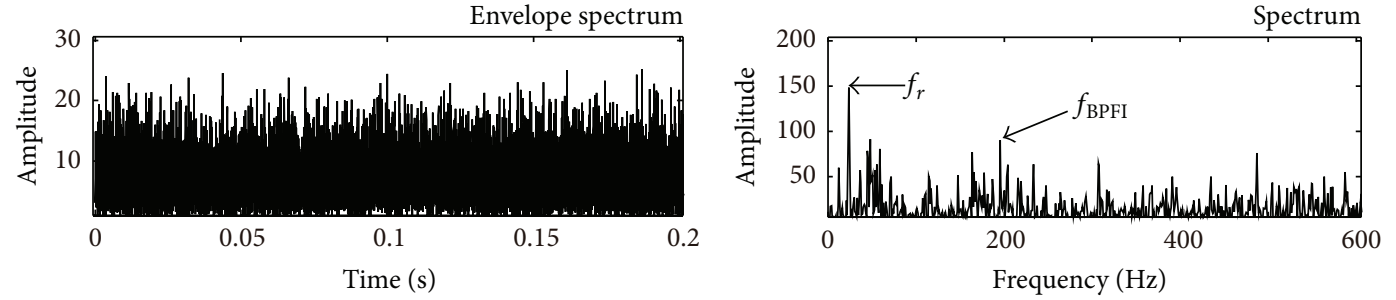

(b)
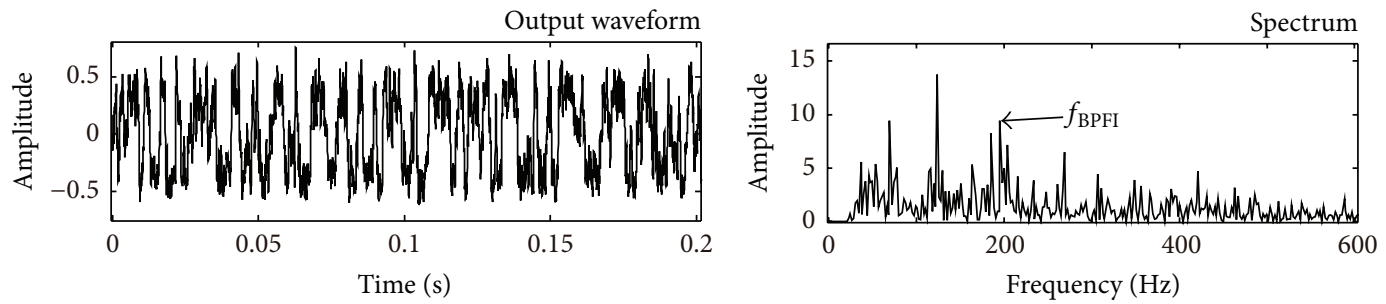

(c)
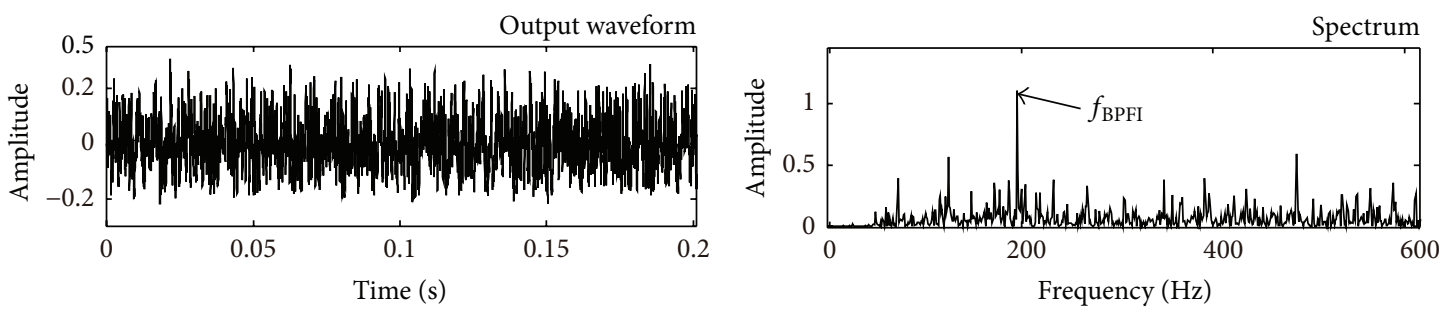

(d)
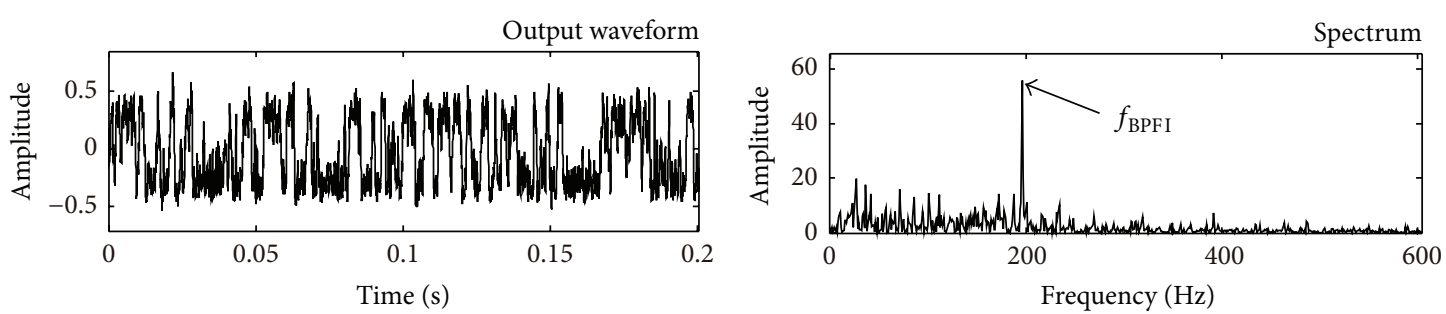

(e)

Figure 14: Optimal outputs of different models with inner-race fault signal: (a) original waveform and its power spectrum, (b) envelope signal and its spectrum filtered with $[500 \mathrm{~Hz}$ and $2500 \mathrm{~Hz}]$, (c) output of bistable SR model and its spectrum with $=0.8, b=38,(\mathrm{~d})$ output of WSGSR model and its power spectrum with $V_{1}=10, R_{1}=0.5, a=0.1$, and $V_{2}=0$ (WSSR), and (e) output of WSGSR model and its power spectrum with $V_{1}=12, R_{1}=1, a=0.2, V_{2}=1$, and $R_{2}=0.45$.

by $V_{2}$ and $R_{2}$, the proposed potential model can easily change between monostable (WSSR) and bistable and has different features. This makes the system more perfect and has a large scope to tune the parameters when optimizing the SR system. The results also show that with the working of potential barrier, output SNR gets higher sometimes especially with heavier noise. It has a stronger ability in filtrating the characteristic frequency ignoring the components of lower or higher frequency. This may owe to the barrier that can weaken the uncorrelated components which can make the particle oscillate more easily without it. 
(4) We have mentioned that when considering the amplitude of output spectrum in Figures 9, 13, and 14, we can easily find that the amplitude of driving frequency in WSGSR is more than 10 times larger than that of the WSSR $\left(V_{2}=0\right)$ even the traditional bistable SR which may be the result of the existence of potential barrier. The higher amplitude means the higher energy on the output signal. So the WSGSR not only keeps the advantages of WSSR but also offers high output energy on driving frequency as the traditional bistable SR model which might benefit to the area of energy harvest. What is more is that we improve the WSSR model to a bistable one where many theories of bistable SR can be applied to, for example, the Kramer's rate. The general type offers us the possibility to have further research on the specific role that the potential shape plays on the performance of SR output signal.

(5) In engineering applications, the key is how to determine the model parameters. However, for an unknown system, we cannot obtain the fault samples in advance. In this case, we know in most of the practical applications that the fault information cannot be obtained easily before the diagnosis work. When processing the signal with band-pass filter and HT, we might not distinguish the fault frequency effectively due to heavy background noise. Then with just a SR model whose parameters are preset, we can extract the component which might not be so distinct. But with this information, we can use the component to adjust the parameters to the optimal combination according to the highest SNR which will make the fault frequency the clearest. If the component is exactly the periodic fault signal, it can be amplified to a high level by the optimal parameters but not vice versa. So the proposed method can offer us a more effective approach to make an accurate and reliable diagnosis.

However, the response of the WSGSR system is still complex and sometimes comes up with randomness as the acquired signal and noise always contain the uncertainty. Hence, a small variation of parameters may result in a quite different output like butterfly effect. What is more is that the model needs some mathematical analysis to support the certain effects that parameters' work has on the output. A deeper mechanism of the WSGSR from both the mathematical and the physical aspects should be investigated. These further studies will make the parameter selection and optimization more effective and gain a more satisfying output.

\section{Conclusions}

An improved potential model of WSGP is investigated to realize the SR effect instead of the traditional bistable one. The model of WSGSR with a particular form of WSSR $\left(V_{2}=0\right)$ has been described in detail to explain its superior performance in bearing fault diagnosis. A diagnostic scheme is present with tuning the system's five parameters. Different from the traditional bistable SR, the features of potential model are affected by the parameters independently. For example, $V_{1}$ works on the well depth, $R_{1}$ works on the well width, $a$ and $R_{2}$ affect the steepness of potential walls, and $V_{2}$ affects the barrier height. It means that an optimal state can be obtained by varying the parameters. During the searching process, a method to confirm the reference frequency to calculate SNR is put forward. The performance of the proposed method has been evaluated by both the simulated and the engineering bearing fault signals. The results among different models show a more efficient and continuous particle motion in WSGP benefiting from its distinct structure. Besides, the WSGSR is insensitive to the noise and owns the better capacity in detecting weak impulse signals and filtrating characteristic frequency with a higher output SNR under different noise and driving frequency levels. The potential barrier also makes it a better performance especially with heavier noise than WSSR. And hence, the driving frequency can be enhanced extremely while the noise interference can be suppressed. As a more general form, the proposed WSGSR offers a good performance in weak periodic signal amplification to diagnose bearing faults. It is reasonable to believe that this method can be used in mechanical diagnostic work especially when the acquired signal is seriously embedded with heavy background noises to amplify the weak periodic signal of fault or characteristic frequency.

\section{Conflict of Interests}

The authors declare that there is no conflict of interests regarding the publication of this paper.

\section{Acknowledgments}

This work was supported in part by the National Natural Science Foundation of China with Grant nos. 51075379 and 11274300. The authors also would like to thank the anonymous reviewers for their valuable comments and suggestions.

\section{References}

[1] R. Benzi, A. Sutera, and A. Vulpiani, "The mechanism of stochastic resonance," Journal of Physics A: Mathematical and General, vol. 14, no. 11, pp. L453-L457, 1981.

[2] L. Gammaitoni, P. Hänggi, P. Jung, and F. Marchesoni, "Stochastic resonance," Reviews of Modern Physics, vol. 70, no. 1, pp. 223287,1998

[3] Y. G. Leng, Y. S. Leng, T. Y. Wang, and Y. Guo, "Numerical analysis and engineering application of large parameter stochastic resonance," Journal of Sound and Vibration, vol. 292, no. 3-5, pp. 788-801, 2006.

[4] J. Tan, X. Chen, J. Wang et al., "Study of frequency-shifted and re-scaling stochastic resonance and its application to fault diagnosis," Mechanical Systems and Signal Processing, vol. 23, no. 3, pp. 811-822, 2009.

[5] M. Lin and Y. M. Huang, "Modulation and demodulation for detecting weak periodic signal of stochastic resonance," Acta Physica Sinica, vol. 55, no. 7, pp. 3277-3282, 2006.

[6] J. Li, X. Chen, and Z. He, "Multi-stable stochastic resonance and its application research on mechanical fault diagnosis," Journal of Sound and Vibration, vol. 332, no. 22, pp. 5999-6015, 2013. 
[7] Q. He, J. Wang, Y. Liu, D. Dai, and F. Kong, "Multiscale noise tuning of stochastic resonance for enhanced fault diagnosis in rotating machines," Mechanical Systems and Signal Processing, vol. 28, pp. 443-457, 2012.

[8] Q. He and J. Wang, "Effects of multiscale noise tuning on stochastic resonance for weak signal detection," Digital Signal Processing, vol. 22, no. 4, pp. 614-621, 2012.

[9] X. Zhang, N. Hu, L. Hu, and Z. Cheng, "Multi-scale bistable stochastic resonance array: a novel weak signal detection method and application in machine fault diagnosis," Science China Technological Sciences, vol. 56, no. 9, pp. 2115-2123, 2013.

[10] N. Tandon and A. Choudhury, "Review of vibration and acoustic measurement methods for the detection of defects in rolling element bearings," Tribology International, vol. 32, no. 8, pp. 469-480, 1999.

[11] J. Lee, F. Wu, W. Zhao, M. Ghaffari, L. Liao, and D. Siegel, "Prognostics and health management design for rotary machinery systems-Reviews, methodology and applications," Mechanical Systems and Signal Processing, vol. 42, no. 1-2, pp. 314-334, 2014.

[12] S. Lu, Q. He, F. Hu, and F. Kong, "Sequential multiscale noise tuning stochastic resonance for train bearing fault diagnosis in an embedded system," IEEE Transactions on Instrumentation and Measurement, vol. 63, pp. 106-116, 2014.

[13] X. Zhang, N. Hu, Z. Cheng, and L. Hu, "Enhanced detection of rolling element bearing fault based on stochastic resonance," Chinese Journal of Mechanical Engineering, vol. 25, no. 6, pp. 1287-1297, 2012.

[14] L. Qiang, W. Taiyong, L. Yonggang, W. Wei, and W. Guofeng, "Engineering signal processing based on adaptive step-changed stochastic resonance," Mechanical Systems and Signal Processing, vol. 21, no. 5, pp. 2267-2279, 2007.

[15] H. L. He, T. Y. Wang, Y. G. Leng, Y. Zhang, and Q. Li, "Study on non-linear filter characteristic and engineering application of cascaded bistable stochastic resonance system," Mechanical Systems and Signal Processing, vol. 21, no. 7, pp. 2740-2749, 2007.

[16] S. Lu, Q. He, and F. Kong, "Stochastic resonance with WoodsSaxon potential for rolling element bearing fault diagnosis," Mechanical Systems and Signal Processing, vol. 45, pp. 488-503, 2014.

[17] J. I. Deza, R. R. Deza, and H. S. Wio, "Wide-spectrum energy harvesting out of colored Lévy-like fluctuations, by monostable piezoelectric transducers," EPL, vol. 100, no. 3, Article ID 38001, 2012.

[18] M. J. Roberts, "Energy moments and cross sections for a gaussian potential," Proceedings of the Physical Society, vol. 82, no. 4, article 319, pp. 594-604, 1963.

[19] J. Adamowski, M. Sobkowicz, B. Szafran, and S. Bednarek, "Electron pair in a Gaussian confining potential," Physical Review B, vol. 62, pp. 13233-13233, 2000.

[20] W. Xie, "Two interacting electrons in a Gaussian confining potential quantum dot," Solid State Communications, vol. 127, no. 5, pp. 401-405, 2003.

[21] G. Stephenson, "Eigenvalues of the Schrödinger equation with a Gaussian potential," Journal of Physics A: Mathematical and General, vol. 10, no. 12, pp. L229-L232, 1977.

[22] M. Cohen, "On the Schrödinger equation with a Gaussian potential," Journal of Physics A: Mathematical and General, vol. 17, no. 3, pp. L101-L104, 1984.

[23] R. D. Woods and D. S. Saxon, "Diffuse surface optical model for nucleon-nuclei scattering," Physical Review, vol. 95, no. 2, pp. 577-578, 1954.
[24] A. K. S. Jardine, D. Lin, and D. Banjevic, "A review on machinery diagnostics and prognostics implementing condition-based maintenance," Mechanical Systems and Signal Processing, vol. 20, no. 7, pp. 1483-1510, 2006. 


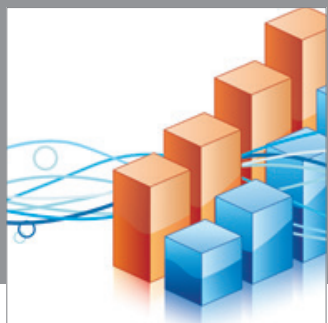

Advances in

Operations Research

mansans

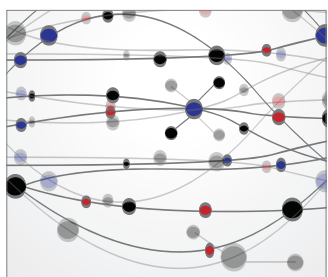

The Scientific World Journal
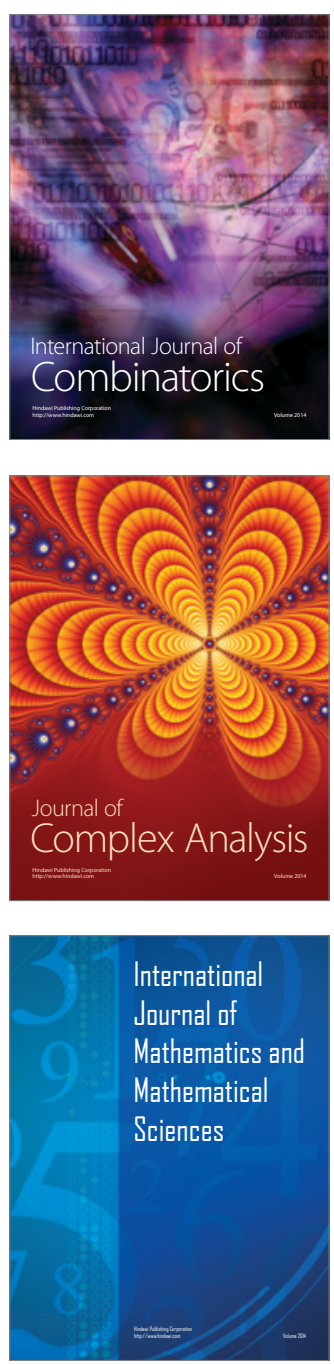
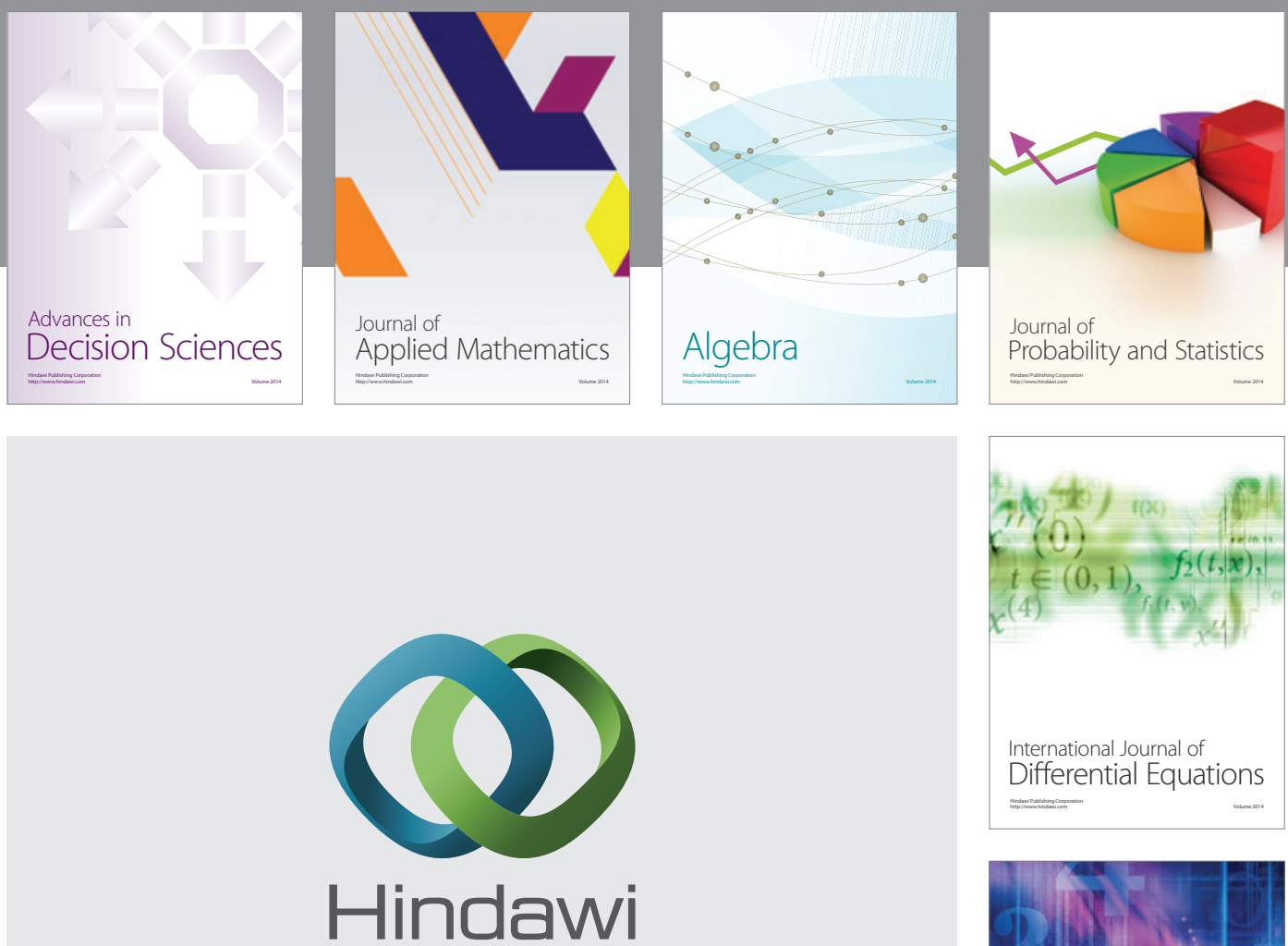

Submit your manuscripts at http://www.hindawi.com
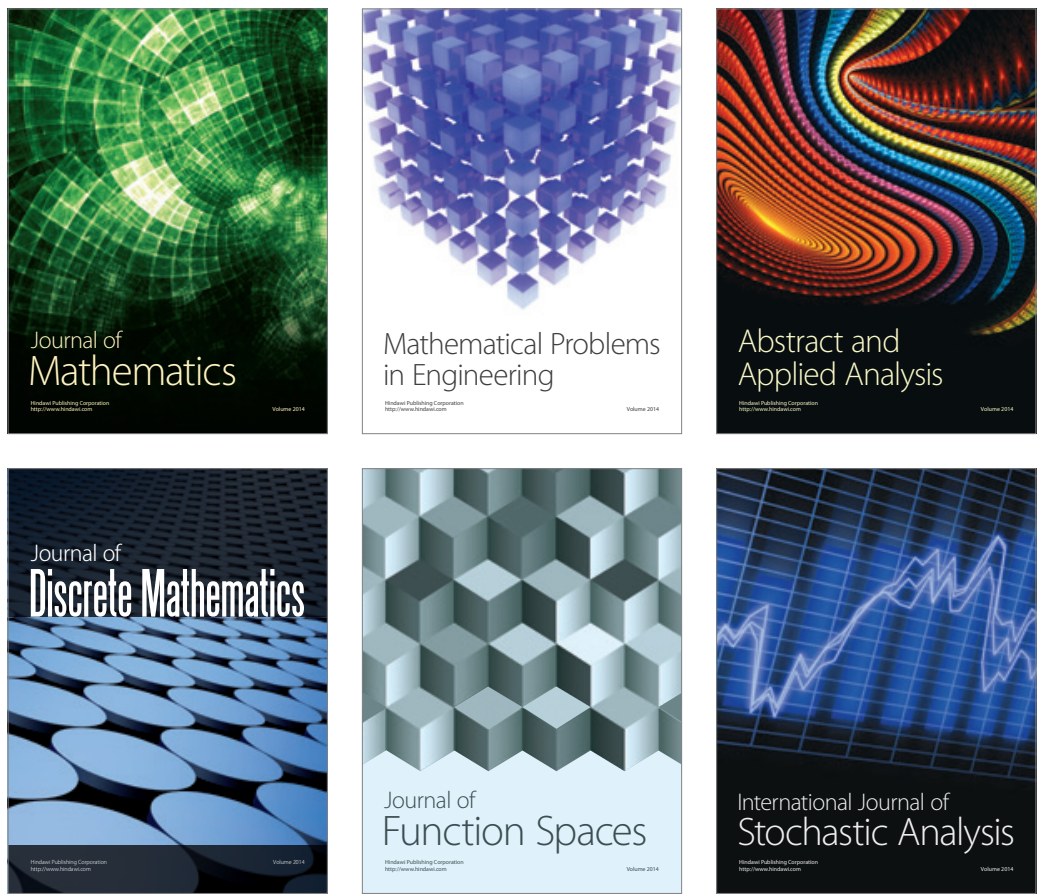

Journal of

Function Spaces

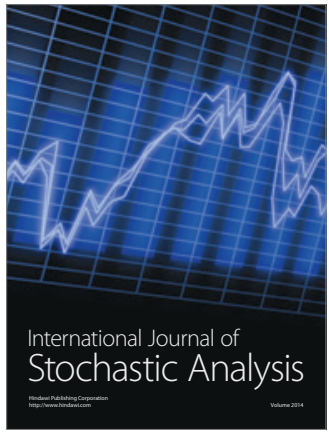

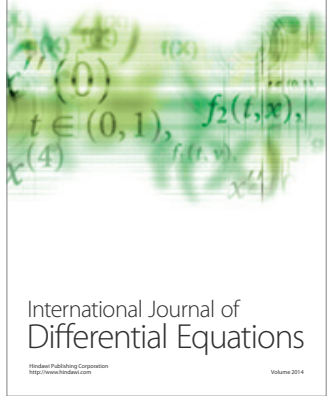
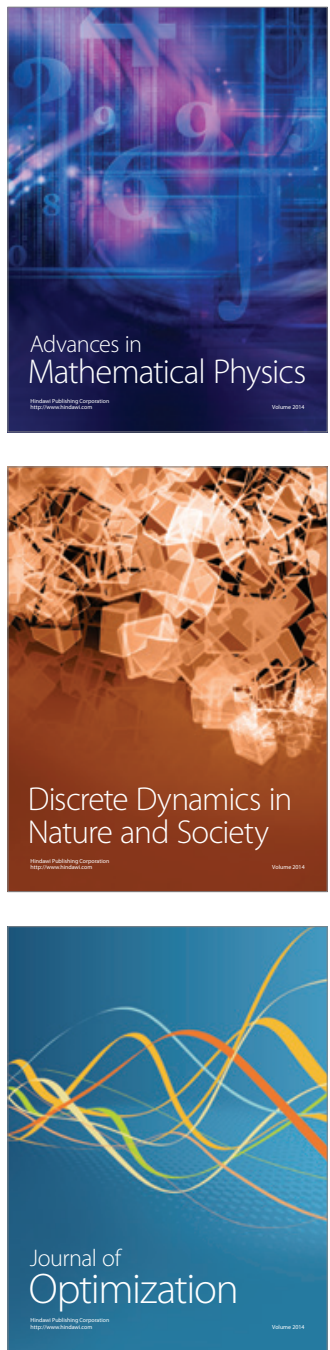